\title{
Distance determination for RAVE stars using stellar models
}

\section{Most likely values assuming a standard stellar evolution scenario}

\author{
T. Zwitter ${ }^{1,2}$, G. Matijevič ${ }^{1}$, M. A. Breddels ${ }^{3}$, M. C. Smith ${ }^{3,4}$, A. Helmi ${ }^{3}$, U. Munari ${ }^{5}$, O. Bienaymé6 ${ }^{6}$ J. Binney ${ }^{7}$, \\ J. Bland-Hawthorn ${ }^{8}$, C. Boeche ${ }^{9}$, A. G. A. Brown ${ }^{10}$, R. Campbel1 ${ }^{11}$, K. C. Freeman ${ }^{12}$, J. Fulbright ${ }^{13}$, \\ B. Gibson ${ }^{14}$, G. Gilmore ${ }^{15}$, E. K. Grebel ${ }^{16}$, J. F. Navarro ${ }^{17}$, Q. A. Parker ${ }^{18}$, G. M. Seabroke ${ }^{19}$, A. Siebert ${ }^{6}$, \\ A. Siviero ${ }^{5,9}$, M. Steinmetz ${ }^{9}$, F. G. Watson ${ }^{20}$, M. Williams ${ }^{9}$, and R. F. G. Wyse ${ }^{13}$ \\ ${ }^{1}$ University of Ljubljana, Faculty of Mathematics and Physics, Ljubljana, Slovenia \\ e-mail: tomaz.zwitter@fmf.uni-lj.si \\ 2 Center of excellence SPACE-SI, Ljubljana, Slovenia \\ 3 Kapteyn Astronomical Institute, University of Groningen, Groningen, The Netherlands \\ 4 Kavli Institute for Astronomy and Astrophysics, Peking University, Beijing, PR China \\ 5 INAF Astronomical Observatory of Padova, 36012 Asiago (VI), Italy \\ ${ }^{6}$ Observatoire de Strasbourg, Strasbourg, France \\ 7 Rudolf Peierls Centre for Theoretical Physics, University of Oxford, UK \\ 8 Sydney Institute for Astronomy, School of Physics, University of Sydney, NSW 2006, Australia \\ 9 Astrophysikalisches Institut Potsdam, Potsdam, Germany \\ ${ }^{10}$ Leiden Observatory, Leiden University, Leiden, The Netherlands \\ 11 Western Kentucky University, Bowling Green, Kentucky, USA \\ 12 RSAA, Australian National University, Canberra, Australia \\ 13 John Hopkins University, Baltimore, Maryland, USA \\ 14 University of Central Lancashire, Preston, UK \\ 15 Institute of Astronomy, Cambridge, UK \\ 16 ARI, Zentrum für Astronomie der Universität Heidelberg, Heidelberg, Germany \\ 17 University of Victoria, Victoria, Canada \\ 18 Macquarie University, Sydney, Australia \\ 19 e2v Centre for Electronic Imaging, Planetary and Space Sciences Research Institute, The Open University, Milton Keynes, UK \\ 20 Anglo Australian Observatory, Sydney, Australia
}

Received 3 May 2010 / Accepted 26 June 2010

\section{ABSTRACT}

The RAdial Velocity Experiment (RAVE) is a spectroscopic survey of the Milky Way which already collected over 400000 spectra of $\sim 330000$ different stars. We use the subsample of spectra with spectroscopically determined values of stellar parameters to determine the distances to these stars. The list currently contains 235064 high quality spectra which show no peculiarities and belong to 210872 different stars. The numbers will grow as the RAVE survey progresses. The public version of the catalog will be made available through the CDS services along with the ongoing RAVE public data releases.

The distances are determined with a method based on the work by Breddels et al. (2010, A\&A, 511, A16). Here we assume that the star undergoes a standard stellar evolution and that its spectrum shows no peculiarities. The refinements include: the use of either of the three isochrone sets, a better account of the stellar ages and masses, use of more realistic errors of stellar parameter values, and application to a larger dataset. The derived distances of both dwarfs and giants match within $\sim 21 \%$ to the astrometric distances of Hipparcos stars and to the distances of observed members of open and globular clusters. Multiple observations of a fraction of RAVE stars show that repeatability of the derived distances is even better, with half of the objects showing a distance scatter of $\lesssim 11 \%$.

RAVE dwarfs are $300 \mathrm{pc}$ from the Sun, and giants are at distances of 1 to $2 \mathrm{kpc}$, and up to $10 \mathrm{kpc}$. This places the RAVE dataset between the more local Geneva-Copenhagen survey and the more distant and fainter SDSS sample. As such it is ideal to address some of the fundamental questions of Galactic structure and evolution in the pre-Gaia era. Individual applications are left to separate papers, here we show that the full 6-dimensional information on position and velocity is accurate enough to discuss the vertical structure and kinematic properties of the thin and thick disks.

Key words. stars: distances - catalogs - surveys - Galaxy: structure - Galaxy: stellar content - stars: statistics

\section{Introduction}

It is now widely accepted that our Galaxy can be used as a testbed to study galaxy formation and evolution (Freeman \& Bland-Hawthorn 2002). A vital tool for this goal is the ability to use complete information on position, motion and physical

* The catalog is only available in electronic form at the CDS via anonymous ftp to cdsarc.u-strasbg.fr $(130.79 .128 .5)$ or via http://cdsarc.u-strasbg.fr/viz-bin/qcat?J/A+A/522/A54 properties of a large number of individual stars. Some of the quantities are usually known, i.e. the sky coordinates and in our case proper motions. But characterizing the three-dimensional position requires knowledge of the distance to the star and describing its motion requires knowledge of its radial velocity. So one needs to measure also these quantities in order to have complete information of an object's place in the 6-dimensional phase space which combines the position and velocity vectors. RAVE (RAdial Velocity Experiment) is the only large scale 
survey measuring radial velocities spectroscopically, with the exception of the SDSS survey which mainly samples the halo of the Galaxy. RAVE already collected over 400000 spectra of 330 000 different stars. Most of the RAVE objects are thin and thick disk stars. So the SDSS and RAVE surveys are complementary in the sample surveyed (halo vs. disk), magnitude range (fainter vs. brighter) and even the celestial hemisphere (North vs. South). The RAVE survey is described in the articles accompanying the first two public data releases (Steinmetz et al. 2006; Zwitter et al. 2008, hereafter Z08). The article describing the third public release is in preparation (Siebert et al. 2010). With the exception of the first data release the subsequent ones also include results of spectroscopic measurements of stellar physical parameters: the effective temperature, surface gravity, and metallicity.

The RAVE dataset can be used to study a range of scientific topics (Steinmetz 2003), including present structure and formation of the Galaxy, remnants of recent accretion events, and discovery of individual peculiar objects and spectroscopic binary stars (Matijevič et al. 2010). It was already used to better constrain the Galactic escape speed at the Solar radius (Smith et al. 2007). Veltz et al. (2008) studied kinematics towards the Galactic poles and identified discontinuities that separate thin disk, thick disk and a hotter component. Seabroke et al. (2008) searched for in-falling stellar streams on to the local Milky Way, and Siebert et al. (2008) estimated the tilt of the stellar velocity ellipsoid and its implications for mass models. Pasetto et al. (in prep.) studied the kinematics of the thick disk. Munari et al. (2008) showed that a diffuse interstellar band at $8620 \AA$ can be used to reliably measure the amount of galactic interstellar reddening. Munari et al. (2009) studied the properties of Luminous Blue Variables in the Large Magellanic Cloud. They used a photo-ionization analysis of their rich emission line spectra to demonstrate the great diagnostic potential of RAVE in spectroscopy of peculiar stars. Additional ongoing studies have been listed in Steinmetz et al. (2006).

Most of these studies would profit from the knowledge of stellar distance. With very few exceptions, RAVE stars are too far away and too faint to have trigonometric parallaxes measured by the Hipparcos mission. The RAVE sample is magnitude limited $\left(9<I_{\text {DENIS }}<13\right)$ but otherwise entirely randomly selected from the Tycho (bright stars), SuperCOSMOS (faint stars) and DENIS catalogs. So only a small fraction of objects observed by RAVE are members of stellar clusters or are of special type for which standard candle techniques can be used to judge their distances. One choice is to assume that all RAVE stars belong to the main sequence and to use their photometric colors to infer their distance (Klement et al. 2008). Unfortunately, giants form about half of the RAVE sample, so any conclusions based on the assumption that they are main sequence stars will be biased. Another possibility is to use photometric colors to isolate the red clump stars. This is a viable technique, but it applies only to a fraction of the sample and may be influenced by contaminating outliers of similar colors.

Over $90 \%$ of the RAVE spectra belong to normal stars and have no substantial instrumental problems. So one may use the knowledge of spectroscopically measured physical parameters to infer their abolute position on the H-R diagram and thus their absolute magnitude. The choice of a near infrared passband $\left(J_{2 \text { MASS }}\right)$ and the fact that the vast majority of RAVE stars lie at high Galactic latitudes $\left(|b|>20^{\circ}\right)$ means that the influence of interstellar reddening is negligible. This approach was introduced by our first paper on the subject (Breddels et al. 2010, hereafter B10). Here we build on this solid foundation and refine this approach by a better accounting for measurement errors, stellar evolution knowledge, allowing for three different sets of stellar isochrone computations, a refined computation method, and by expanding the sample to the recently observed stars. Another distance computation method using a Bayesian approach tested on a Galactic pseudo-data sample will also be used to calculate distances to RAVE stars (Burnett \& Binney 2010).

All these efforts are aimed at a common goal to derive the most reliable distances to RAVE stars. The goal is important and it is likely that different distance determination methods will be preferred for different types of stars. The situation could indeed prove to resemble the problem of computation of stellar isochrones where it is best for the user to choose a particular set depending on the problem which is being considered.

By the end of the decade a public catalog of extremely accurate and numerous astrometric distances measured by the ESA's mission Gaia is expected to become available. Still, as with any magnitude limited survey, there will be a significant portion of stars for which their distances are too large for accurate trigonometric parallaxes to be determined. So photometric distance determination methods will remain to be useful also in the postGaia era of Galactic astronomy.

The structure of the paper is as follows: Sect. 2 introduces the refined distance determination method. In Sect. 3 we test the results by comparing them to the available Hipparcos trigonometric parallax measurements and to the distances of open clusters with some confirmed members observed by RAVE. Section 4 discusses statistical properties of the results and the structure of the publicly available catalog, to be followed by general conclusions in Sect. 5. The reader is encouraged to consult B10 for a more complete discussion of different methods to measure stellar distances and some of the scientific results. Detailed scientific results related to the structure and evolution of the Galaxy as well as distances of individual stars and stellar clusters will be published separately.

\section{Refined distance determination method}

B10 used values of stellar parameters and the Yonsei-Yale (Demarque et al. 2004) isochrones to derive the absolute $J_{2 \text { MASS }}$ magnitudes. Comparison with the apparent 2MASS $J$ magnitudes then yielded distances. The values of stellar parameters measured by RAVE are not error-free. B10 assumed constant errors in temperature $(300 \mathrm{~K})$, gravity $(0.3 \mathrm{dex})$, and metallicity $(0.25 \mathrm{dex})$ for all stars. So the values derived by RAVE were jittered according to these assumed standard uncertainties. 5000 realisations of each observation were calculated by sampling Gaussian distributions in each observable. The final absolute $J$ magnitude was the mean of the absolute $\mathrm{J}$ magnitudes pertaining to the closest isochrone matches for each of the realisations. The closest match was derived by minimizing the usual $\chi^{2}$ statistics. The distribution of absolute $J$ magnitudes for individual realisations also gave the standard error of the derived absolute magnitude and thus of the distance to the star.

In formulating refinements to the B10 approach we alter the method for distance derivation. Instead of using the closest match, an expectation value of the absolute magnitude is derived considering several isochrones at a given metallicity. Our goal is to derive the most likely distance to the star. So we assume that we are dealing with a normal star that follows stellar evolution as mirrored by theoretical isochrones. We use our knowledge of stellar evolution to compensate for some of the errors in values of stellar parameters as derived by RAVE. So the results of this paper should be very useful in Galactic and general population 
studies. But we note that our results may not be applicable to peculiar objects or to those in rapid transit phases of stellar evolution. The latter because the adjacent longer lived and so more populated evolutionary phases may be within the measurement errors. The refinements to the B10 method are described below.

\subsection{The three sets of isochrones}

The distance computation of B10 was based on the Yonsei-Yale (hereafter YY, Demarque et al. 2004) set of isochrones. Here we add two additional sets: the Dartmouth (Dotter et al. 2008) and the Padova isochrones (Bertelli et al. 2008). While the former two sets are very useful for the derivation of properties of stars which are on or close to the main sequence, their computations do not extend to the asymptotic giant branch (AGB) or red clump (RC) evolutionary phases. The Padova set on the other hand nicely covers both the AGB and the RC phase. So it is particularly useful to derive distances to giant stars. All sets of isochrones tabulate also the absolute $J$ magnitude. In the case of YY and Dartmouth sets the absolute $J$ magnitude can be directly compared to the apparent one measured by the 2MASS survey, while Eq. (1) from Koen et al. (2007) was used to transform the Padova set into the 2MASS system. In all cases we used isochrones without any enhancement of alpha elements. This quantity is not measurable reliably by RAVE and has a very small influence on the final distance results (see B10, for a detailed discussion).

All isochrone sets cover the parameter space in the necessary detail and in some cases even offer interpolation routines to derive values at points in between. These routines were used when possible, otherwise a standard linear interpolation scheme was used. So we generated a fine grid of isochrones with age, mass and metallicity steps much smaller than the observational errors. The mass step was always smaller than $0.01 M_{\odot}$, and the metallicity step was 0.1 dex. Sampling in age is discussed below.

All computations were performed three times, deriving a separate set of distances for each set of isochrones. We publish all three distances and their errors. It is then up to the user to decide which one is the most suitable for the type of problem or the type of object that is being considered. Details of isochrone computation and the corresponding assumptions are described in detail in the original papers presenting each isochrone set.

\subsection{Isochrones uniformly spaced in time}

B10 used a set of isochrones with 40 different ages, spaced logarithmically between 0.01 and 15.0 Gyr. This implies a time step of only 2 Myr at the young end and up to $2.5 \mathrm{Gyr}$ at the old end of the isochrone set. Such a choice covers the [temperature, gravity] plane well. But the use of the closest match implies an implausibly high probability that a certain realisation lies within a rapid evolutionary phase, such as a contraction towards the main sequence or a transit from the main sequence towards the region of giants. The bulk of the Galactic populations is expected to be found in slow, long-lasting phases of stellar evolution. So, although it may be possible that RAVE observed some objects in rapid phases of stellar evolution, this is not probable in individual cases.

In the present calculations the isochrones are spaced uniformly and not logarithmically in time. So the density of grid points is inversely proportional to the speed of motion of a star across the [temperature, gravity] plane. We use a step of 20 Myr for ages up to 12 Gyr. The youngest age depends on its availability for a particular isochrone set. It is $0.1 \mathrm{Gyr}$ for the YY set, 1 Gyr for Dartmouth and 0.5 Gyr for the Padova one. Uniform spacing in time gives a proper weight to longer evolutionary phases, while rapid transit periods have their importance diminished. The adopted age range includes the span of ages of a general stellar population as determined by the GenevaCopenhagen survey (Holmberg et al. 2009).

\subsection{Weighting according to mass and absolute magnitude}

The quantity changing along a theoretical isochrone is stellar mass. Isochrones are tabulated so that connecting adjacent points follows their shape well. So the points are tabulated in relatively large mass steps while the isochrone lies on the main sequence. The mass steps get much smaller when the isochrone bends toward giants.

We use a scheme that weights individual solutions. Clearly the weight of a given point should be proportional to a mass step $\mathrm{d} m$ which is an average of mass differences to the adjacent mass points on a given isochrone. It should also be noted that high mass stars are much less frequent than ones with sub-solar masses. $\mathrm{d} N / \mathrm{d} m$ is the Galactic mass function as given in Eq. (17) of Chabrier (2003) for sub-solar stars and in his Eq. (11) for stars more massive than the Sun. Moreover, more luminous stars at a given apparent magnitude are farther away than their less luminous counterparts. The volume of space within which the stars with a given absolute magnitude $M_{I}$ in the Cousin $I$ band appear brighter than the given apparent limiting magnitude is proportional to $10^{-0.6 M_{I}}$. We use the $I$ band, as both the surveys used to select the observing sample and the final RAVE spectra are centered on this band. If we assume that the space density of stars does not depend on distance the following relative weight $x$ of points along the isochrone is obtained:

$x=\mathrm{d} m(\mathrm{~d} N / \mathrm{d} m) 10^{-0.6 M_{I}}$.

One may argue that the $\mathrm{d} N / \mathrm{d} m$ term can be used only for a volume limited survey, while RAVE is a magnitude limited one. Similarly, the stellar distribution is not uniform. Stars at large distances are quite far away from the Galactic plane, so the frequency of these luminous, massive stars is even lower than would be in the case of a uniform space density. Fortunately these assumptions are not crucial. It is reasonable to take into account that massive stars are uncommon and that RAVE is able to sample a larger volume of more luminous stars. But the exact mathematical form of these prescriptions is not so important, and we can choose the simplest relation using varying weights. The reason lies in the quality of RAVE's measurement of values of stellar parameters. Their errors are rather small, even though not negligible. So any large offsets in temperature, gravity and metallicity are virtually excluded. The role of Eq. (1) is to ascribe a relative weight to points on the isochrones that are close to the initial RAVE values.

As a test we recalculated the whole database using $x=\mathrm{d} m$. So we ignored any mass or luminosity dependence. The results are illustrated in Fig. 1. We see that most stars are closer than in the case when we use Eq. (1). The magnitude term that favours more luminous and hence more distant stars in Eq. (1) therefore dominates over the mass function $\mathrm{d} N / \mathrm{d} m$ which disfavours massive (and so luminous) stars. But differences in distances derived by the test procedure and the usual one are small: the mode of the distance ratio distribution derived by the two methods is 0.94 , and the median is 0.87 . So even the extreme simplification which ignores any assumptions used to derive Eq. (1) changes the distances by only $\sim 10 \%$. 


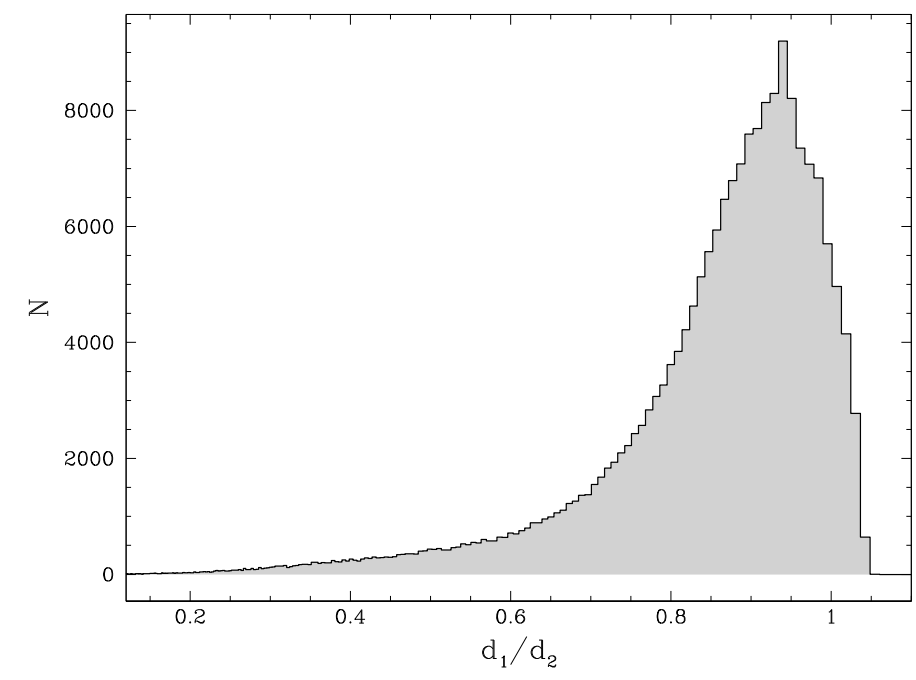

Fig. 1. Ratio of distances derived under different assumptions. $d_{1}$ is derived assuming that the probability of a star to be observed does not depend on its mass or brightness, while the distance $d_{2}$ takes into account the mass and brightness functions, as described in the text.

\subsection{Assumed errors of stellar parameters}

The errors of stellar parameter values measured by RAVE depend on stellar type. Typical temperature errors for stars cooler than $9000 \mathrm{~K}$ are around $400 \mathrm{~K}$. The errors are smallest for supergiants. Their atmospheres are the most transparent ones, so the wealth of spectral lines arising at different optical depths can improve the temperature accuracy. Understandably the errors for metal-poor stars are larger than for their Solar counterparts. The errors get considerably worse for stars hotter than $9000 \mathrm{~K}$, where most metal lines are missing and the spectrum is largely dominated by hydrogen lines. Similarly, strong wings of hydrogen lines, which are sensitive to gravity, allow small gravity errors in hot stars. On the other hand rather narrow metallic lines in the RAVE wavelength range, including those of Ca II, do not allow an accurate determination of gravity in cool stars. The gravity error in cool stars has a strong gravity dependence: in dwarfs it is $\sim 0.6 \mathrm{dex}$, but the rather transparent atmospheres of giant stars still allow for a more accurate gravity determination, with a scatter that can be as low as 0.2 dex. Finally, the typical error in metallicity for stars cooler than $7000 \mathrm{~K}$ is 0.2 dex. The error for hotter stars is understandably much larger, as these stars lack most of the metallic lines in their spectra.

Given this vivid behaviour it seems important to allow for a variation of the accuracy of stellar parameter values across the parameter space. This is a refinement over other studies aiming at distance computation using values of stellar parameters as determined by RAVE. Here the errors are obtained from a linear interpolation of results presented in Fig. 19 and Eqs. (22) and (23) of Z08. Note that these error estimates may be rather conservative (Steinmetz et al. 2009).

\subsection{Calculating the distance modulus}

Values of stellar parameters, as determined by RAVE, are not error-free. So B10 first determine the closest match to the RAVE values in the isochrone parameter space. This closest match is then jittered according to the assumed parameter uncertainties. Each of the realizations again yields the closest match in the isochrone space, and a suitable average of these closest matches yields the final value of the distance and its scatter.
This procedure can benefit from a refinement regarding the use of the closest match. Imagine we have a Solar type mainsequence star that would have the reported values of RAVE parameters placing it just above the main sequence of the H-R diagram. Jittering the reported value of its gravity would mean that half of the realizations would imply a higher gravity and so a main-sequence solution. But the other half of the jittered realizations would claim the star is above the main sequence in a rapid transition towards the giant phase. The use of the closest matches would thus produce half of the realizations involving massive stars in a rapid transit toward giants and half of mainsequence cases. The relative weight of realizations involving the rapid transition phase is therefore too large. It also influences the value of the mean of absolute $J$ magnitudes for individual realizations and so the derived distance to the star.

We use a modified procedure to calculate the most likely value of the absolute $J$ magnitude. The distance modulus then follows from its difference with the apparent $J$ magnitude as measured by the 2MASS survey. We do not jitter the measured parameter values and calculate individual realizations to be solved with the closest match. Instead, the expectation value of the absolute magnitude is derived using the relative weights

$$
\begin{aligned}
& X(a, m)=x(a, m) \mathrm{e}^{-y(a, m)} \\
& y(a, m)=\frac{[T(\mathrm{RAVE})-T(a, m)]^{2}}{2 \sigma_{T(\mathrm{RAVE})}^{2}}+\frac{[\log g(\mathrm{RAVE})-\log g(a, m)]^{2}}{2 \sigma_{\log g(\mathrm{RAVE})}^{2}}
\end{aligned}
$$

where $a$ is age and $m$ mass at a given point on the isochrone with the metallicity value of the RAVE measurement. Standard deviations of the measured value of temperature $\left(\sigma_{T \text { (RAVE) }}\right)$ and gravity $\left(\sigma_{\log g(\mathrm{RAVE})}\right)$ are determined as described in Sect. 2.4. The final value of the absolute $J$ magnitude $\left(M_{J}\right)$ and its standard deviation $\left(\sigma\left(M_{J}\right)\right)$ are calculated from the first moments of the distribution

$$
\begin{aligned}
& M_{J}=\frac{\sum_{a} \sum_{m} M_{J}(a, m) X(a, m)}{\sum_{a} \sum_{m} X(a, m)} \\
& \sigma^{2}\left(M_{J}\right)=\frac{\sum_{a} \sum_{m} X(a, m)\left[M_{J}-M_{J}(a, m)\right]^{2}}{\sum_{a} \sum_{m} X(a, m)} .
\end{aligned}
$$

The procedure makes a brute force average of all solutions for an isochrone set of a metallicity measured by RAVE. Typical values of metallicity errors are $\simeq 0.2 \mathrm{dex}$, or about half of the step in a grid of stellar atmosphere models used by Z08 (their Fig. 19). On the other hand the errors in temperature or gravity are typically larger than the grid step. The isochrone sets for different values of metallicity are very similar, as already discussed by B10 (see its Fig. 2). A lower metallicity corresponds to a more transparent stellar atmosphere with a hotter effective temperature, but has little effect on the stellar energy output. So a metallicity change causes a shift of the isochrone in the temperature direction, but the absolute magnitude stays very similar (see isochrone comparison in Fig. 2b). It is thus not surprizing that the results on absolute magnitude show no significant changes when using metallicity as another independent variable. In particular, the distances do not change by more than $10 \%$ for $90 \%$ of the spectra when using metallicity as an independent variable. But an extra term significantly prolongs the calculation. So we decide to adopt the simplified form given in Eq. (2). If isochrones with the value of RAVE-measured metallicity do not exist they are obtained by a linear interpolation from the adjacent ones. Similarly, some of the isochrone sets are calculated for a set of iron abundances $([\mathrm{Fe} / \mathrm{H}])$ which in general differ from the 

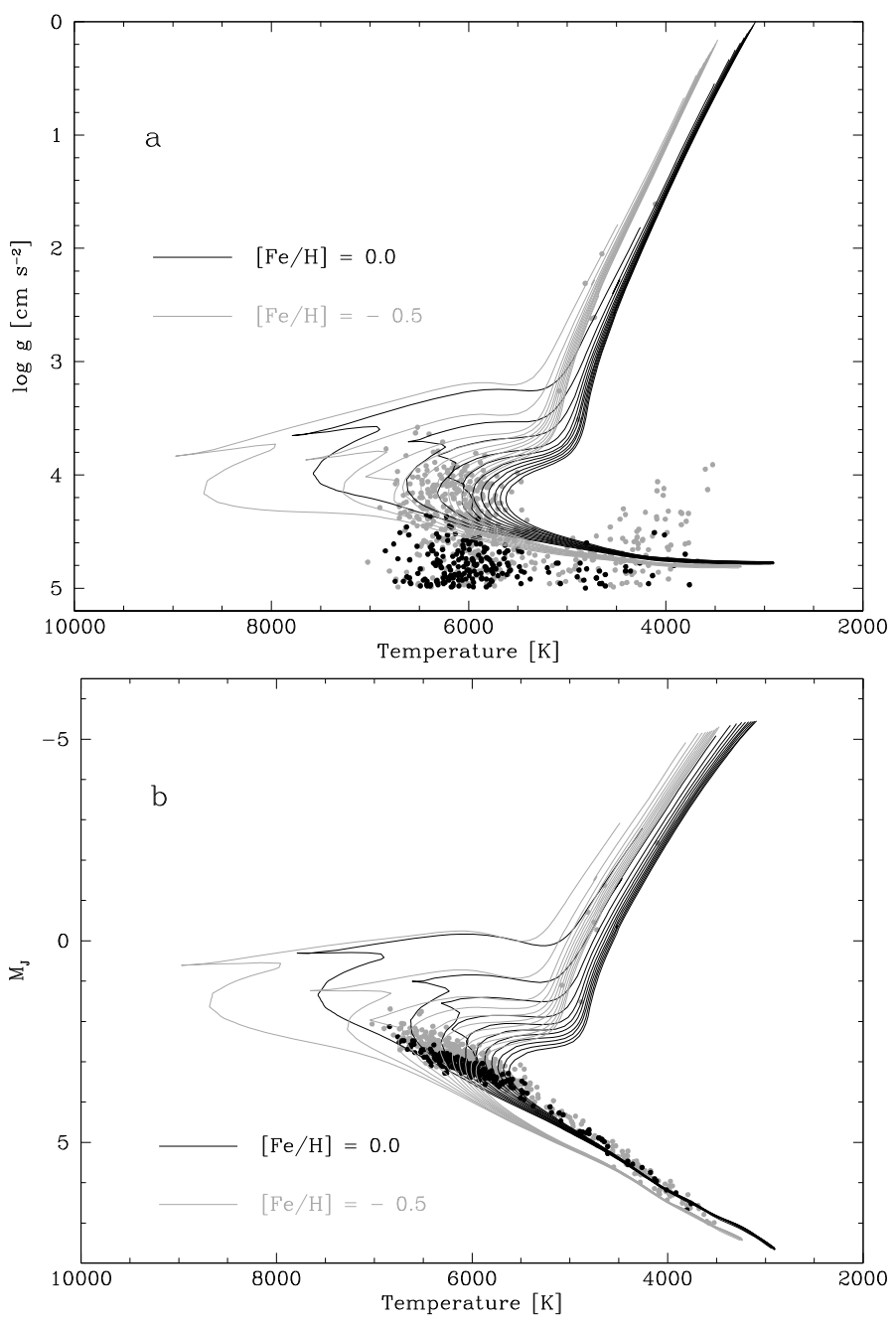

Fig. 2. HR diagram of Hipparcos stars closer than 130 pc. The top panel a) shows RAVE measurements of gravity and effective temperature, while the bottom panel $\mathbf{b}$ ) presents the result of our calculation of the absolute $J$ magnitude using the YY set of isochrones. Grey points are Hipparcos stars with a trigonometric distance error smaller than $20 \%$, a spectroscopic distance error below $32 \%$ and with RAVE spectra with $S / N>40$. Black points are the subset where the B10 and the three isochrone set calculations presented in this paper found a spectroscopic distance error smaller than $32 \%$. Black and gray lines trace YY isochrones with Solar and subsolar composition and for ages between 1 and $11 \mathrm{Gyr}$ with a $1 \mathrm{Gyr}$ spacing. The vast majority of Hipparcos stars close enough to have accurate trigonometric parallaxes are dwarfs.

metallicities $([\mathrm{M} / \mathrm{H}])$ measured by RAVE. Equation (21) from $\mathrm{Z} 08$ is used to convert between iron abundance and metallicity. Similarly we use Eq. (20) from Z08 to calibrate the metallicity values.

We explained in Sect. 2.2 that the isochrones are uniformly spaced in age, while Sect. 2.3 discusses relative weights $x(a, m)$ along an isochrone. A Gaussian form of Eq. (2) means that only points on the isochrones that have the temperature and gravity within a few standard deviations from the RAVE measurement are important. This scheme weights each solution according to its distance from the position measured by RAVE. So the weighting goes by distance in the [temperature, gravity] parameter space and not by area, as is the case with the closest match. So we avoid giving excessive weights to isochrones in the scarcely populated regions of the parameter space, where a given solution is the closest match to a substantial range of the [temperature, gravity] pairs.

\section{Tests of the derived distances}

Stellar distances are generally not known, so we can test our results only for certain subsets of the measured sample. Two such cases will be considered: Hipparcos stars and confirmed members of stellar clusters. In future we plan to test the results also with a Galactic pseudo-data set (Burnett \& Binney 2010). Combined results of these tests and experience obtained through a scientific use of distance results will be discussed in future releases of RAVE data.

\subsection{Hipparcos stars}

Hipparcos stars have their distances measured through trigonometric parallaxes (van Leeuwen 2007). Our goal is to use objects with well determined trigonometric distances and with highquality RAVE spectra. This choice can then allow us to check for systematic errors in our distance computation which is the primary goal of our test. The formal errors on the parallaxes are $1.0 \pm 0.5$ mas, so we adopted a lower limit of 7.7 mas for the parallax, corresponding to a distance limit of $130 \mathrm{pc}$. Moreover, for this test we considered only RAVE spectra with a $S / N$ ratio $>40$. RAVE observed 1525 such stars so far. Finally we require that a formal error in spectroscopic distance modulus $<0.6$ mag (corresponding to a distance error of $32 \%$ ). The last constraint is satisfied by 725 spectra for calculations based on the YY isochrones, 776 spectra for the Dartmouth set, and 674 for the Padova set. 277 of them had their spectroscopic distances already included in B10.

RAVE confirmed that most of these stars have a Solar-like composition $([\mathrm{M} / \mathrm{H}]=-0.12 \pm 0.25)$. Figure $2 \mathrm{a}$ shows RAVE measurements of the effective temperature and surface gravity. As expected nearly all stars are dwarfs. The magnitude limits of the RAVE survey also imply that most stars are somewhat hotter (and brighter) than the Sun, lying in the general turn-off region. As discussed in Sect. 2.4 their temperature determination is rather accurate, but their gravity errors are quite high. So RAVE is able to determine their general main-sequence type, but not any further details. The error on gravity is even worse for cool stars, so the small group of deviant stars at the bottom-right corner of Fig. 2a can easily be normal main-sequence stars.

The top panel of Fig. 2 presents the input for the spectroscopic distance calculation, while the bottom panel depicts its output. The ordinate is now the calculated absolute $J$ magnitude resulting from the YY set of isochrones. We see that the method nicely corrects the too-high gravities from the top panel and places the sub-dwarf stars back on the main sequence. Also the cool stars supposedly lying above the main sequence are now nicely settled onto it.

Figure 3 compares trigonometric distances derived by Hipparcos $\left(d_{\varpi}\right)$ to the RAVE spectroscopic distances $(d)$ as determined by $\mathrm{B} 10$ and by the present calculation using the three isochrone sets. Our restriction to very high-quality measurements of trigonometric parallax allows us to ignore any transformation bias due to the non-linear relation between parallax and distance.

The spectroscopic distance determinations are generally in good agreement with their trigonometric parallax values. Table 1 reports the best fits of the relation $d=k d_{\omega}$ for two samples and for four different sets of calculations. The slopes $k$ are close to 
Table 1. Comparison of spectroscopic and trigonometric distances ${ }^{(1)}$.

\begin{tabular}{lcccccc}
\hline \hline & \multicolumn{3}{c}{ Sample A } & \multicolumn{3}{c}{ Sample B $^{(3)}$} \\
Calculation & $k$ & $\sigma$ & spectra & $k$ & $\sigma$ & spectra \\
\hline B10 & $0.92 \pm 0.01$ & 0.18 & 277 & $0.89 \pm 0.01$ & 0.18 & 207 \\
This paper (YY isochrones) & $1.03 \pm 0.01$ & 0.23 & 725 & $1.09 \pm 0.02$ & 0.25 & 207 \\
This paper (Dartmouth isochrones) & $1.01 \pm 0.01$ & 0.23 & 776 & $1.07 \pm 0.02$ & 0.24 & 207 \\
This paper (Padova isochrones) & $1.03 \pm 0.01$ & 0.25 & 674 & $1.09 \pm 0.02$ & 0.26 & 207 \\
\hline
\end{tabular}

Notes. (1) Spectroscopic $(d)$ and trigonometric $\left(d_{\varpi}\right)$ distances are assummed to follow the relation $d=k d_{\varpi}$. (2) Hipparcos stars with a trigonometric distance error smaller than 20\%, a spectroscopic distance error below 32\% and with RAVE spectra with $S / N>40$. (3) A subset of Sample A where all four calculations find a spectroscopic distance error smaller than $32 \%$.

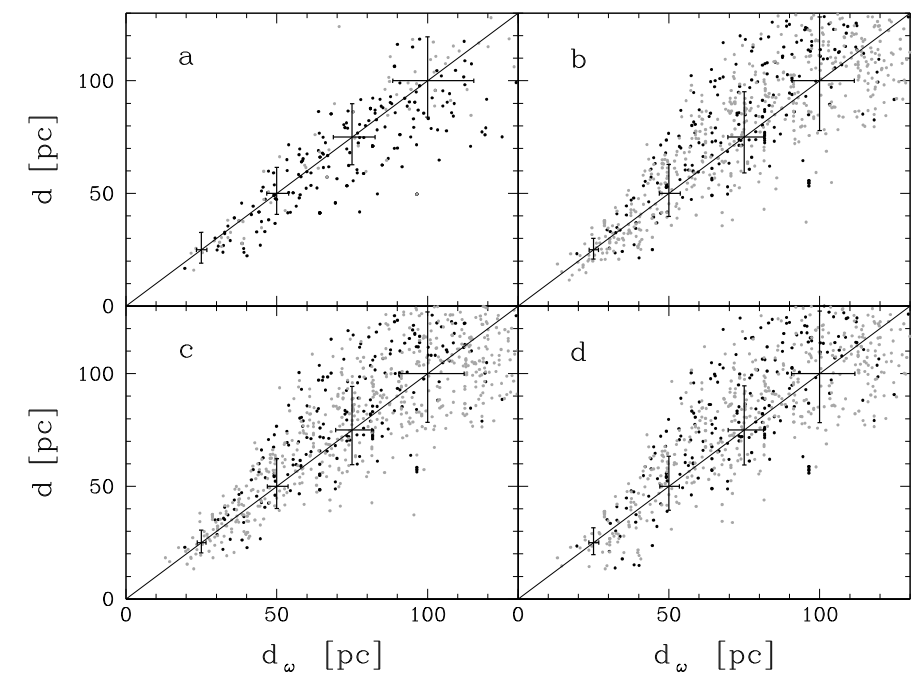

Fig. 3. Comparison of distances to Hipparcos stars. The trigonometric parallax distances $d_{\varpi}$ are compared to spectroscopic distances $d$ from B10 a), and to values derived here using the YY b), Dartmouth $\mathbf{c}$ ), and Padova d) isochrones. We are using the same selection criteria and color scheme as in Fig. 2. The errorbars show average formal errors for stars in four distance bins. The diagonal line is a 1:1 relation.

unity. The B10 distances are somewhat underestimated, while the ones reported in this paper are slightly overestimated if compared to trigonometric parallax measurements. On the other hand the scatter in $k$ is a bit larger for measurements in this paper than was the case in B10. This is partly due to a difference in the assumed errors of stellar parameters. While B10 use the same error for all spectra, our errors are variable and depend on the type of star and the $\mathrm{S} / \mathrm{N}$ ratio of the spectrum. Our assumed errors are generally larger, a typical error in gravity is $0.45 \mathrm{dex}$ compared to 0.3 dex used by B10. Assuming fixed error values from $\mathrm{B} 10$ ( $300 \mathrm{~K}$ in temperature and $0.3 \mathrm{dex}$ in gravity) we get more satisfying fits for the Sample B: $k=1.03 \pm 0.01, \sigma=0.21$ (YY isochrones), $k=1.04 \pm 0.01, \sigma=0.21$ (Dartmouth), and $k=1.05 \pm 0.01, \sigma=0.22$ (Padova set).

Note that many of the Hipparcos stars with the temperature close to $6000 \mathrm{~K}$ have $\log g \sim 4.8$, a value too high for this part of the main sequence (Fig. 2a). This offset challenges the distance routine which assumes a Gaussian error distribution. The offset is more frequent for the Hipparcos stars than for typical RAVE spectra. About $15 \%$ of the Hipparcos stars hotter than $5500 \mathrm{~K}$ have a claimed $\log g>4.8$, compared to just $8 \%$ of the cases for the general RAVE sample. Also, Hipparcos stars sample the cool part of the main sequence down to temperatures of $3500 \mathrm{~K}$. But the general RAVE sample has hardly any dwarfs cooler than $4500 \mathrm{~K}$, with a vast majority at around $6000 \mathrm{~K}$. The difference is due to a magnitude limited nature of the survey, where cool stars are sampled only in a small volume around the Sun.

So the scatter obtained for the Hipparcos stars may not be representative of the entire population of RAVE spectra of the main-sequence stars. Note that the Hipparcos stars are generally brighter than the general sample observed by RAVE, so their spectra have a higher $S / N$ ratio. Thus the distance error estimates for the Hipparcos stars may be somewhat optimistic. It is interesting to note that the smallest distance errors are frequently found for stars with overestimated gravity values. This is understandable, as for such stars the main-sequence solution is an obvious choice. On the other hand the stars with more realistic and so lower values of gravity could also be turn-off stars, so their absolute magnitude determination is less certain.

To summarize, trigonometric parallax measurements and results of our spectroscopic distance computations show average discrepancies of $\$ 5 \%$. The scatter for measurements of individual stars is $\$ 24 \%$. Including the formal error of the Hipparcos parallaxes in quadrature, we find that the spectroscopic distances are accurate to within $\lesssim 22 \%$.

\subsection{Distances to members of star clusters}

RAVE observed virtually no giant stars with an accurate measurement of their trigonometric parallax. Our spectroscopic distance measurement, however, can easily reach the giants that form about half of the RAVE sample. So we used known members of open and globular clusters observed by RAVE to extend our check of the distance scale.

The Vizier catalog of open clusters of Dias et al. (2002), Version 2.8 was used. A star is considered to be a known open cluster member if: (i) it lies within the apparent diameter of the cluster center in both coordinates; (ii) its proper motion and the value for the cluster reported by Dias et al. (2002) do not differ by more than the sum of their reported uncertainties; (iii) the same is true also for radial velocities (if Dias et al. 2002, do not report the radial velocity error a difference of 2 $\mathrm{km} \mathrm{s}^{-1}$ is considered acceptable); (iv) the Simbad catalog reports that the object is a "star in cluster" (class $* \mathrm{iC}$ ). Clearly these requirements are conservative. They exclude many potential cluster members observed by RAVE. But our goal here is to use definite cluster membership to check our distances. We also checked the Harris et al. (1996) catalog of globular clusters. The star J125905.2-705454 is 3.43 arcmin from the center of the globular cluster NGC 4833, which has a halfmass radius of $2.41 \mathrm{arcmin}$. Its heliocentric radial velocity of $202.1 \pm 0.8 \mathrm{~km} \mathrm{~s}^{-1}$ (as measured by RAVE) matches the cluster's radial velocity of $200.2 \pm 1.2 \mathrm{~km} \mathrm{~s}^{-1}$. Similarly its RAVE metallicity $[\mathrm{M} / \mathrm{H}]=-1.2$ is consistent with the cluster iron abundance of $[\mathrm{Fe} / \mathrm{H}]=-1.79$. So we consider it to be a member of 
Table 2. Distances to members of star clusters.

\begin{tabular}{|c|c|c|c|c|c|c|c|c|c|}
\hline \multirow[t]{2}{*}{ Object-ID } & \multirow{2}{*}{$\begin{array}{r}\mathrm{RA}_{\mathrm{J} 2000.0} \\
\text { [deg] }\end{array}$} & \multirow{2}{*}{$\begin{array}{r}\text { Dec }_{\text {J2000.0 }} \\
\text { [deg] }\end{array}$} & \multicolumn{2}{|c|}{ Cluster } & \multirow[t]{2}{*}{$\overline{S / N}$} & \multicolumn{4}{|c|}{ 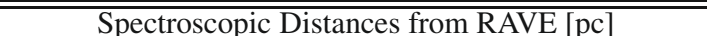 } \\
\hline & & & ID & $d[\mathrm{pc}]$ & & B10 & Yonsei-Yale & Dartmouth & Padova \\
\hline J000128.6-301221 & 0.369417 & 30.205861 & Blanco 1 & 270 & 64 & $260 \pm 80$ & 70 & $260 \pm 60$ & $260 \pm 60$ \\
\hline $\mathrm{JOC}$ & & 22 & o 1 & & 4 & & & 0 & 280 \\
\hline 19 & 0 & 57 & 423 & 770 & 73 & \pm 960 & 20 & 20 & 90 \\
\hline $\mathrm{O}$ & 2 & 00 & NGC 2422 & 45 & 44 & 400 & & 62 & 50 \\
\hline $\mathrm{J} 07$ & 50 & 889 & NGC 2477 & 1300 & 50 & \pm 1200 & 310 & 10 & 116 \\
\hline J07 & 125 & -38.4 & NGC 2477 & 1300 & 61 & $2600 \pm 1300$ & 320 & 20 & 1720 \\
\hline M6 & 50 & 304444 & NGC 2682 & 910 & 89 & & 170 & 70 & 150 \\
\hline M6 & 833 & 51 & NGC 2682 & 910 & 50 & & 280 & 90 & 80 \\
\hline M67 & 875 & 083 & NGC 2682 & 910 & 57 & $2510 \pm 700$ & 260 & 270 & 1010 \\
\hline$(*)$ & 0 & 361 & C 2682 & 910 & 48 & $1900 \pm 940$ & 1000 & 104( & 960 \\
\hline M67 & 083 & 361 & C 2682 & 910 & 99 & $1540 \pm 770$ & 170 & 70 & 190 \\
\hline 5411 & 5000 & -58.488444 & NGC 3532 & 490 & 52 & $640 \pm 270$ & 120 & 20 & 120 \\
\hline 77_2236511 & 165.9 & -58.960972 & NGC 3532 & 490 & 39 & $560 \pm 250$ & \pm 150 & 130 & $450 \pm 150$ \\
\hline 00502_1 & 2250 & -43.164611 & NGC 3680 & 938 & 67 & $2670 \pm 990$ & $1210 \pm 280$ & $=280$ & $1140 \pm 260$ \\
\hline J125905.2-705454 & 194.771667 & -70.914972 & NGC 4833 & 5900 & 37 & & $5500 \pm 1000$ & $5900 \pm 1100$ & $4900 \pm 1100$ \\
\hline
\end{tabular}

Notes. (*) A likely cluster member: Simbad does not list it as a cluster member, but it is within the cluster area and has a radial velocity consistent with the cluster membership.

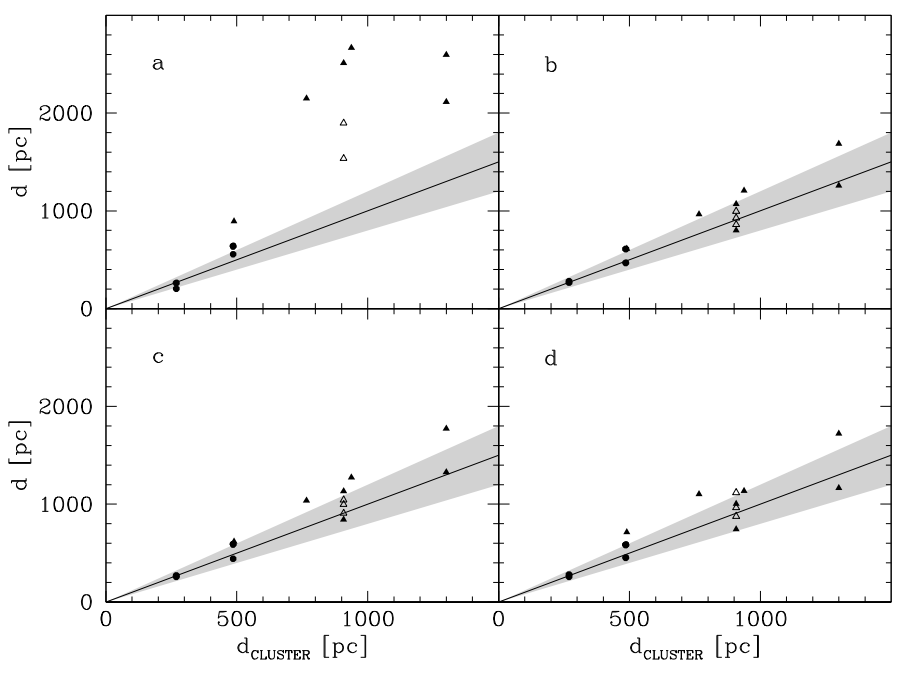

Fig. 4. Distances to open clusters $\left(d_{\text {CLUSTER }}\right)$ from Dias et al. (2002, 2007) compared to spectroscopic distances $d$ of cluster members observed by RAVE from B10 a), and to values derived here using the YY b), Dartmouth c), and Padova d) isochrones. Circles denote dwarfs $\left(\log g_{\text {RAVE }}>3.8\right)$ and triangles likely giants $\left(\log g_{\text {RAVE }} \leq 3.8\right)$. Open symbols mark the likely members of $\mathrm{M} 67$. The line marks the 1:1 relation. The pairs of distances in the shaded area are equal to within $20 \%$.

this globular cluster. No other members of globular clusters were found.

Figure 4 and Table 2 summarize the results. Altogether we have observations of 11 secure members of 7 different open clusters, one globular cluster member and 3 additional likely cluster members. Four stars are dwarfs $(\log g>3.8)$, the rest are giants $(\log g \leq 3.8)$. Our results show a satisfactory performance for dwarfs and for giants. Globular cluster member J125905.2705454 is omitted from Fig. 4 due to scale. Table 2 shows that also for this supergiant $\left(T_{\text {eff }}=4250 \mathrm{~K}, \log g=0.8\right)$ the spectroscopic distance is consistent with the value from the literature. The derived spectroscopic distances are generally within $20 \%$ of the distance to the cluster as given in Dias et al. (2002) and Harris et al. (1996). The average and the scatter of the spectroscopic to cluster distance ratio for the entries in Table 2 equals $0.97 \pm 0.14$ $(1.11 \pm 0.08), 1.03 \pm 0.15(1.16 \pm 0.08)$, and $0.89 \pm 0.15$
$(1.11 \pm 0.08)$ if using the YY, Dartmouth, and Padova isochrones respectively. The values in brackets are obtained if we fit only the open cluster members. The difference of solutions inside and outside the brackets is quite large, given that it corresponds to a contribution of a single globular cluster member. So we note that all values are consistent with the 1:1 relation to within $\sim 10 \%$.

B10 use observations of the open cluster NGC 2682 (M 67) to study the distance scale of giants. The distances found are generally too large. Here we show that our distances are consistent with the adopted value of $910 \mathrm{pc}$ from the literature. The two members in Table 2 give $930 \pm 160$ pc (YY isochrones), $980 \pm 160$ pc (Dartmouth), and $880 \pm 150$ pc (Padova). If we would add the likely M 67 members by their heliocentric radial velocity $\left(31.2 \mathrm{~km} \mathrm{~s}^{-1}<\mathrm{RV}<33.4 \mathrm{~km} \mathrm{~s}^{-1}\right)$ the number of observed M 67 members would increase to five and the results would be $930 \pm 110$ pc (YY), $980 \pm 110$ pc (Dartmouth), and $940 \pm 100$ pc (Padova).

Stellar clusters have a known value of the color excess $E_{B-V}$, so they allow some discussion of the influence of interstellar extinction on our distance estimates. Fiorucci \& Munari (2003) give for the extinction in the $J$ band $A_{J} \simeq 0.887 E_{B-V}$. Nearly all clusters in Table 2 have $E_{B-V}<0.1$, so their extinction in the $J$ band is $A_{J}<0.089$. Ignorance of extinction makes their distances overestimated by no more than $4 \%$. The only clusters with the color excess larger than 0.1 are NGC $2477\left(E_{B-V}=0.24\right.$, Dias et al. 2002) and NGC $4833\left(E_{B-V}=0.33\right.$, Harris et al. 1996). Neglecting interstellar extinction should thus make their distances overestimated by $10 \%$ and $14 \%$, respectively. Note that these two clusters lie very close to the Galactic plane $\left(b=-5.8^{\circ}\right.$ and $b=-8.0^{\circ}$, respectively). A vast majority of the RAVE sample has $|b|>20^{\circ}$, so its interstellar extinction can be safely ignored.

RAVE observed more objects that are likely members of open clusters and satisfy the same selection criteria, with the difference that Simbad does not recognize them as cluster members. There are a number of such likely members of NGC 2423, and a few objects in the open clusters Collinder 121, ASCC 33, and Alessi 5. Here we compared spectroscopic distance measurement of confirmed cluster members to known cluster distances. A study of possible cluster members and applications using spectroscopic distances to stellar clusters will be published elsewhere. 


\section{Derived distances}

The method described in Sect. 2 has been applied to a sample of RAVE stars with spectroscopically determined values of temperature, gravity and metallicity. First we describe the sample and stellar properties as reflected in the derived absolute magnitudes. Then we move to statistical properties of derived distance distributions, including their error estimates. We conclude with a detailed discussion of the catalog and its availability.

\subsection{The sample}

RAVE is an ongoing survey that uses the multi-fiber spectrograph of the UK Schmidt telescope at the Anglo Australian Observatory to obtain stellar spectra with a resolving power of $\sim 7500$ in the wavelength range from $8410 \AA$ to $8795 \AA$. It is conducted by an international collaboration led by M. Steinmetz and aims to obtain a million stellar spectra in the magnitude range $9<I_{\text {DENIS }}<13$. The mode of the signal-to-noise ratio per pixel of the $>400000$ that have so far been gathered follows the relation $S / N=10^{-0.2\left(I_{\text {DENIS }}-19.1\right)}$ (Z08). A detailed description of the survey is given in the papers accompanying the first two public data releases (Steinmetz et al. 2006; Zwitter et al. 2008), soon to be followed by the third one (Siebert et al. 2010).

This paper is based on the sample of 332747 currently reduced spectra with a measurement of radial velocity. For 292261 of these we have determined the values of stellar parameters, with the rest being either too noisy or suffering from systematic problems such as a contamination by the second interference order. A subsample of 251364 have $S / N>20$, so dependable estimates of the values of stellar parameters (temperature, gravity and metallicity), which are virtually free from systematic offsets, could be obtained (Z08). The $S / N$ ratio depends only on the $I_{\text {DENIS }}$ magnitude of the object, on the observing conditions, and on the performance of the instrument. Objects to be observed are magnitude selected and observed in a random order. So our decision to discuss only objects with $S / N>20$ is equivalent to a modified magnitude cut and can be taken into account when discussing statistical properties of the sample. A few percent of the stars turn out to be double-lined spectroscopic binaries, coronal emission objects, or show other peculiarities. Our distance estimates assume the spectrum belongs to a single normal star. So these peculiar objects need to be excluded, for which we used an automated classification routine (Matijevič et al. 2010). The routine unavoidably fails to identify some of the binary stars. In the case of a single-lined binary star the distance would be unaffected. On the other hand the distances to unresolved doublelined spectroscopic binaries are underestimated by up to a factor of $\sqrt{2}$. But such worst cases are rare (Matijevič et al. 2010). If a star in a binary is two magnitudes brighter than its unidentified companion, the error in its estimated distance drops to $8 \%$. The final sample of stars for which the distances have been estimated contains 235064 spectra with $S / N>20$ and without detected peculiarities. They belong to 210872 different stars.

\subsection{Derived absolute magnitudes}

The immediate result of our method is an estimate of the absolute magnitude of the star. We use the $J_{2 \text { MASS }}$ passband because the apparent magnitudes measured by the 2MASS survey are accurate, available for virtually all stars in our sample (Z08, Table 7) and sufficiently red to allow us to ignore any effects of interstellar extinction (see Sect. 3.2).
The properties of the sample can be studied with the $H-R$ diagram in Fig. 5, which shows the results of the absolute magnitude calculations of B 10 and by the method presented here for all three isochrone sets. The relative density of stars is represented by linearly spaced colored contours. A subset of Solar metallicity isochrones is overlaid for illustration purposes.

Two groups of stars are apparent: the moderate temperature stars which are close to the main sequence and the group of cool giants. Cool dwarfs are almost missing. This can be understood, because the RAVE survey has a fairly limited magnitude span $\left(9<I_{\text {DENIS }}<13\right)$, so the volume of space with cool dwarfs of appropriate apparent magnitude is smaller than the one with hotter dwarfs and much smaller than the volume occupied by luminous and thus distant giants. Hot dwarfs are also infrequent, which is not surprising given the low probability of their formation and their short lives. Similar selection effects diminish also the relative frequency of supergiants. As a result of these considerations RAVE observes mostly dwarfs slightly hotter than the Sun and giants with surface temperatures of $\sim 4700 \mathrm{~K}$.

Figure 5a leaves some room for improvement. Many of the main-sequence stars seem to be just leaving the main sequence, which is unlikely. Note that this is even more true if their metallicities were lower than Solar. Also, giants lie above the Solar-metallicity isochrones. This would be fine if they were very metal-poor, but RAVE metallicities tell that they are not. Consequently B10 find that the distance to M67 giants is $1480 \mathrm{pc}$ (if excluding the red clump stars), a value 60\% larger than in the literature. The B10 distances to Hipparcos dwarfs however turn out to be in agreement with astrometric distance determination of van Leeuwen (2007).

Results using the refined method are given in Figs. 5b-d. These panels show the results for the sample of 145028 normal spectra with $S / N>20$ for which B10 also give distances (Fig. 5a). The whole sample we present in our Catalog (Sect. 4.6) is larger and includes 235064 spectra. Both samples are drawn from the same selection strategy and observation protocol, implying very similar errors in derived values of stellar parameters and thus distances. Results of the refined computations show that dwarf stars now lie very close to the main sequence and the giants are in the area of moderately metal-poor giants with an age of a few Gyr. A closer inspection reveals that most mainsequence stars are more luminous than the locus of the zero-age main sequence. This is understandable, as a main-sequence star with $\sim 6000 \mathrm{~K}$ and an age of $\sim 5 \mathrm{Gyr}$ is close to the turn-off point (see the 5 Gyr isochrone in Fig. 5), so it is notably brighter than it was when younger.

Results for the calculations using the YY (Fig. 5b) and Dartmouth (Fig. 5c) isochrones are very similar. However, the graph based on Padova isochrones (Fig. 5d) shows a notable concentration of giants at $M_{J} \simeq-0.9$. This difference arises because only Padova isochrones include a proper treatment of the redclump region. Since stars spend a long time in this region we expect many of the giants observed by RAVE to lie in the red clump. Hence, distances based on the Padova isochrones are to be preferred over ones calculated using the YY or Dartmouth isochrones, at least for giants. The locus of the red-clump stars lies at a bit hotter temperatures than the RGB stars with the same absolute magnitude. So the red-clump stars in Fig. 5d are not superimposed on the red-giant branch.

The red clump region in Fig. $5 \mathrm{~d}$ can be studied further. Figure 6 a shows a histogram of $M_{J}$ for temperatures between $4500 \mathrm{~K}$ and $5000 \mathrm{~K}$. Note the pronounced red clump maximum, modelled as a Gaussian with the peak at $M_{J}=-0.91 \pm$ 0.02 and $\sigma=0.18$. The parameters were adjusted so that the 


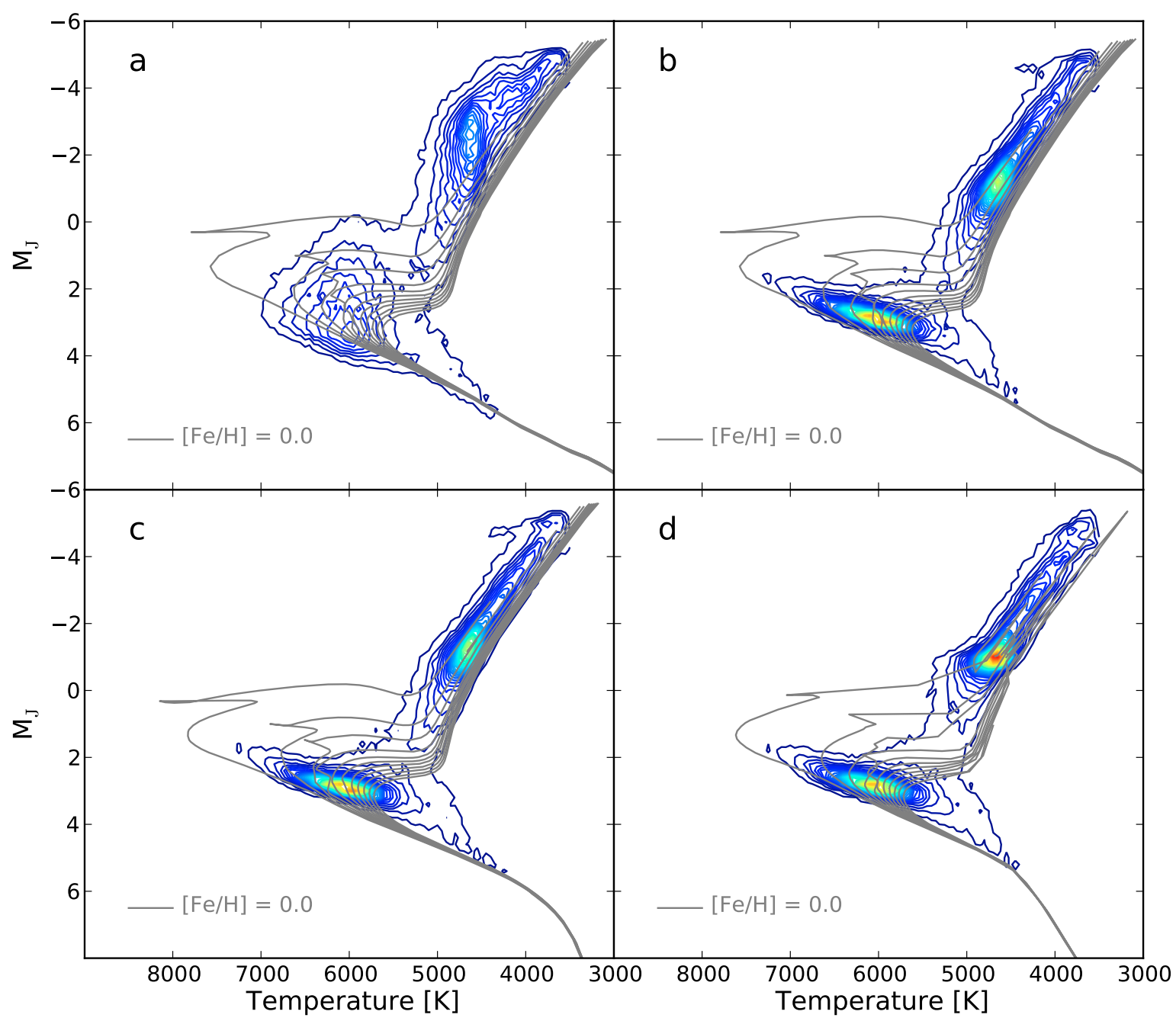

Fig. 5. Frequency of occurence of solutions in the absolute $J$ magnitude vs. temperature diagram for four sets of calculations: a) results from the B10 paper, b-d) new calculations using the YY, Dartmouth and Padova isochrones, respectively. All panels include the same set of 145028 spectra common to all four calculations, but the appearance of $\mathbf{b}-\mathbf{d}$ ) for the whole dataset of 235064 stars would be almost identical. Fifty colored ison-frequency contours are linearly spaced between 20 and 1900 stars per bin with a step of 40 . Each bin spans $50 \mathrm{~K}$ in the temperature and $0.2 \mathrm{mag}$ in the magnitude direction. Gray lines trace Solar composition isochrones between 1 and $11 \mathrm{Gyr}$ with a $1 \mathrm{Gyr}$ spacing.

underlying contribution of the RGB stars remains a smooth function of the absolute magnitude. RGB stars contribute $49 \%$ of all RAVE spectra with $-1.5<M_{J}<-0.5$ and $4500 \mathrm{~K}<$ $T_{\text {eff }}<5000 \mathrm{~K}$. If the absolute magnitude interval is broadened to $-3<M_{J}<1$ the fraction rises to $62 \%$. So their contribution should be accounted for when selecting the red clump stars by their effective temperature, colour or absolute magnitude.

The 2MASS survey reports also the $K$ magnitudes for the same stars. The histogram using the same selection criteria (Fig. 6b) can again be deconvolved into a smooth RGB contribution and a Gaussian red clump maximum with the peak at $M_{K}=-1.61 \pm 0.02$ and $\sigma=0.19$. RGB stars contribute $45 \%$ of all RAVE spectra with $-2.1<M_{K}<-1.1$ and $4500 \mathrm{~K}<$ $T_{\text {eff }}<5000 \mathrm{~K}$. If the absolute magnitude interval is broadened to $-3<M_{K}<1$ the fraction rises to $60 \%$.

The position of the red clump peak is in agreement with the values frequently used in the literature. Valentini \& Munari (2010) used a carefully selected sample of red clump stars to estimate $M_{K}=-1.55$ and $M_{J}=-0.87$. Similarly Van Helshoecht \& Groenewegen (2007) give $M_{K}=-1.57 \pm 0.05$ and Groenewegen (2008) derives $M_{K}=-1.54 \pm 0.04$.

\subsection{Formal errors of derived distances}

The errors in our distances arise from four sources. First, the values of temperature, gravity and metallicity as derived by RAVE have their uncertainties (see Sect. 2.4). Moreover, the isochrone solution can be uncertain due to intersections of isochrones in the parameter space. Also the isochrone positions themselves depend on assumptions used in their calculation. Finally, we convert the absolute $M_{J}$ magnitude into distance assuming a negligible interstellar absorption and a reliable measurement of the apparent magnitude by the 2MASS survey. The last point has been already discussed and generally does not present a major concern. We tried to compensate for isochrone differences by performing all calculations three times, each time with a different set of isochrones. The uniqueness of the solution has been discussed in detail by B10. This leaves us with studying the influence of the errors of measured stellar parameters on the derived distances.

Figure 7 plots the formal errors of the absolute magnitude and distance, both as a histogram and as a cumulative plot. The results are very similar when using YY and Dartmouth 

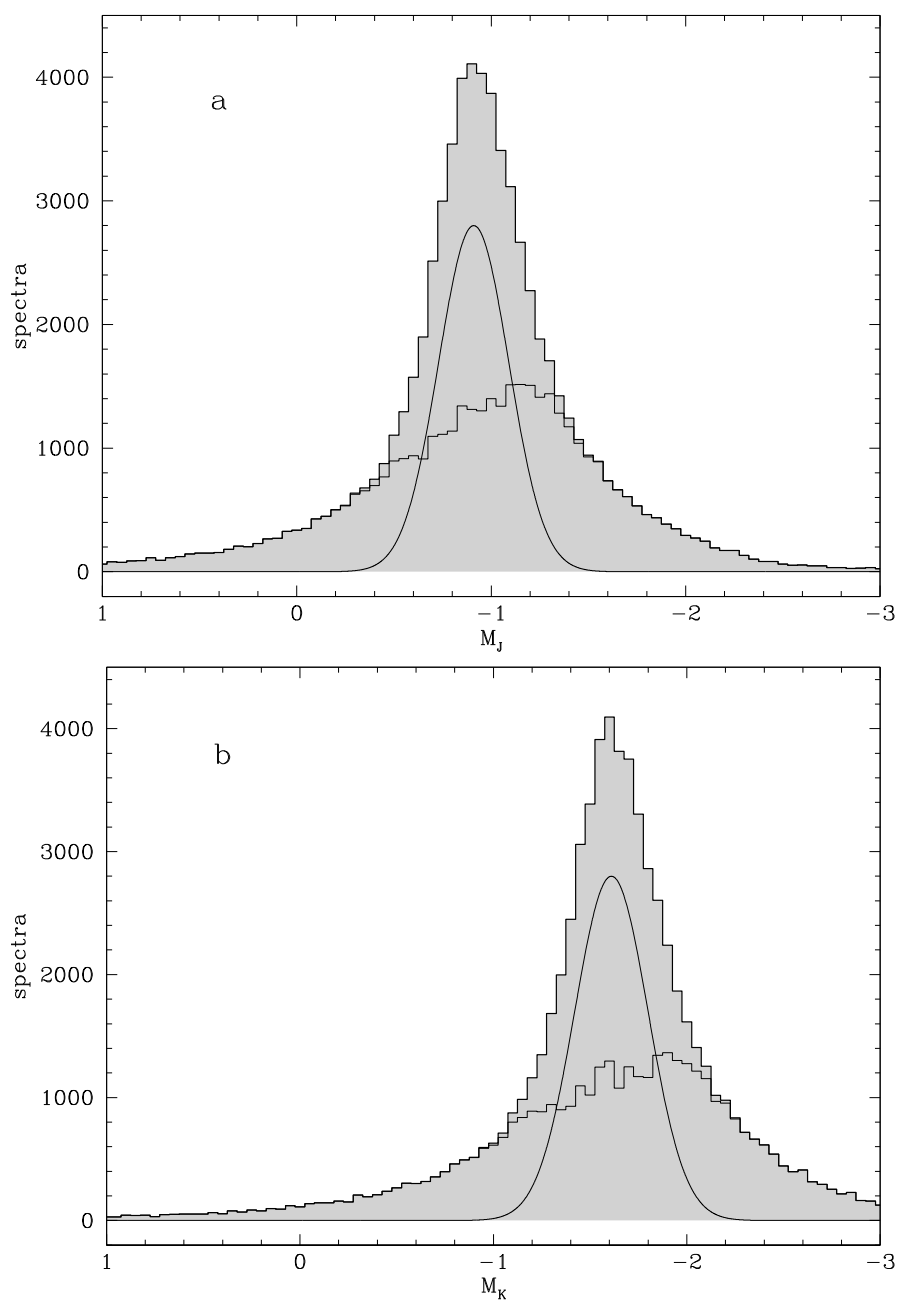

Fig. 6. Histogram of absolute $J$ (panel a) and $K$ (panel b) magnitudes for red clump stars. 2MASS $J$ and $K$ apparent magnitudes were combined with the distance calculation using the Padova isochrone set to derive the absolute $J$ and $K$ magnitudes. Red clump stars are assumed to have $4500 \mathrm{~K}<T_{\text {eff }}<5000 \mathrm{~K}$. So the top panel presents a histogram of $M_{J}$ values in Fig. $5 \mathrm{~d}$ between these two temperatures. The histogram representing all stars in this temperature interval is decomposed into an assummed Gaussian red clump peak and a smooth red giant branch contribution. For parameter values see text.

isochrones, while the Padova set yields marginally smaller errors. The reason is a stronger concentration of stars in the red clump region. There are virtually no stars with distance errors smaller than $10 \%$, which is understandable. On the other hand half of the stars have their distances determined to better than $33 \%$. These results are based on the formal errors obtained from Eq. (4) and the errors in the parameter described in Sect. 2.4.

The errors of the stellar parameters vary across the $H-R$ diagram, so it should be the same also with the errors of derived distances. The specific structure of the RAVE sample, which has a negligible number of very young stars still settling on the main sequence and which lacks large numbers of high-mass mainsequence stars, implies that the absolute magnitude $M_{J}$ could be used to label the position of the star on the $H-R$ diagram. In effect this means that we can use the vertical coordinate in Fig. 5 as an independent coordinate for studying distance errors over the $H-R$ diagram. Figure 8 plots the formal scatter of the absolute $J$ magnitude for different luminosity classes. The thick line traces the median error and the shaded area marks the $20 \%$ to
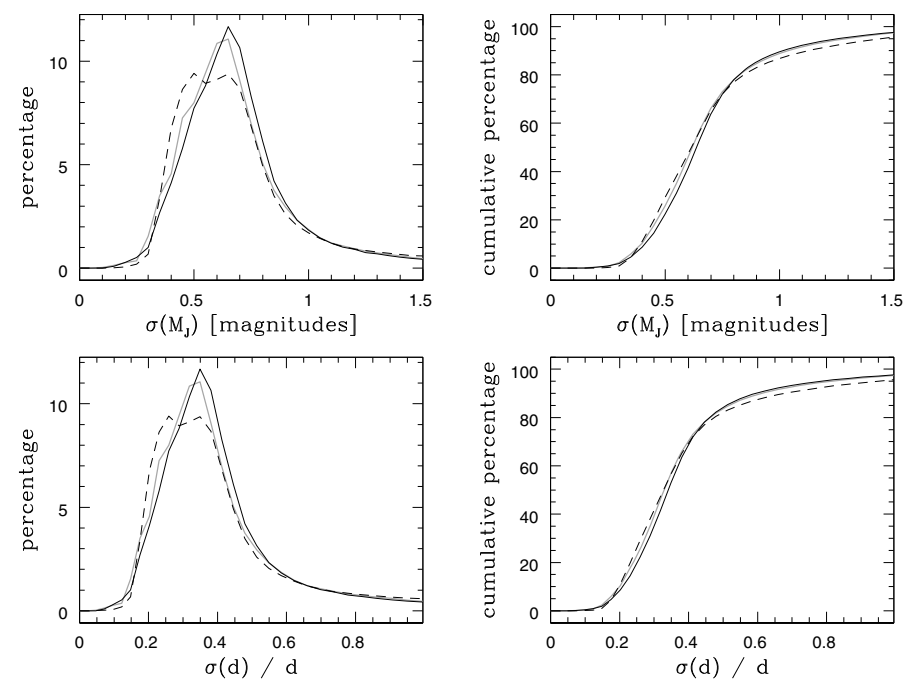

Fig. 7. Formal error distribution histogram (left) and cumulative plot (right) for absolute $J$ magnitude (top) and distance (bottom). Black lines are for YY isochrones, grey ones for Dartmouth, and the dashed ones for the Padova ones. The histograms have bin widths of 0.05 mag and include 235064 spectra that have $S / N>20$ and do not show any peculiarities.

$80 \%$ percentiles of the error distribution. The dashed line shows the relative number of objects per absolute magnitude bin. It is encouraging to note that the distance errors are lowest for stars on the main sequence and in the red clump, i.e. the most frequently occurring objects in our sample. On the other hand the errors are the largest for stars with $M_{J} \sim 1.0$, i.e. for stars that are just leaving the main sequence. This is understandable as this is the region in which isochrones of different ages intersect in the (temperature, gravity) plane, making it possible to find a wide variety of possible solutions. Another region with large uncertainties is that occupied by cool main-sequence stars. Here the errors are large on account of uncertain values of the gravity from RAVE (see Sect. 2.4). For some of these stars RAVE measures gravities that are too low, so the stars are placed above the main sequence (see Fig. 2). Thus both the main-sequence and giant solutions enter Eq. (4) and contribute to an increased scatter. Fortunately these regions of increased distance scatter are sparsely populated in our sample.

Stellar distances are derived using either of the three isochrone sets. These sets are not identical, so it is interesting to compare the results. Figure 9 shows the scatter of the three absolute magnitude values derived for each spectrum. Absolute magnitudes for the upper main sequence $\left(T_{\text {eff }} \simeq 6500 \mathrm{~K}, \log g \simeq 4.0\right.$ ) and red clump ( $T_{\text {eff }} \simeq 4750 \mathrm{~K}, \log g \simeq 2.5$ ) are almost identical for all isochrone sets. At the same time these regions have the largest number of stars in our sample (Fig. 5). On the other hand the most discordant results are for stars in the transition zone between the main sequence and giants. Here some isochrone sets favour the main-sequence and others the giant solution. The dispersion is somewhat elevated also for stars more luminous than the red clump $(\log g \simeq 1.9)$. The origin of this discrepancy lies in differences in the physics included in computations of individual isochrone sets. Only the Padova set allows for the red clump, so the distances computed with this set are to be preferred for giants. 
T. Zwitter et al.: Distance determination for RAVE stars using stellar models. II.

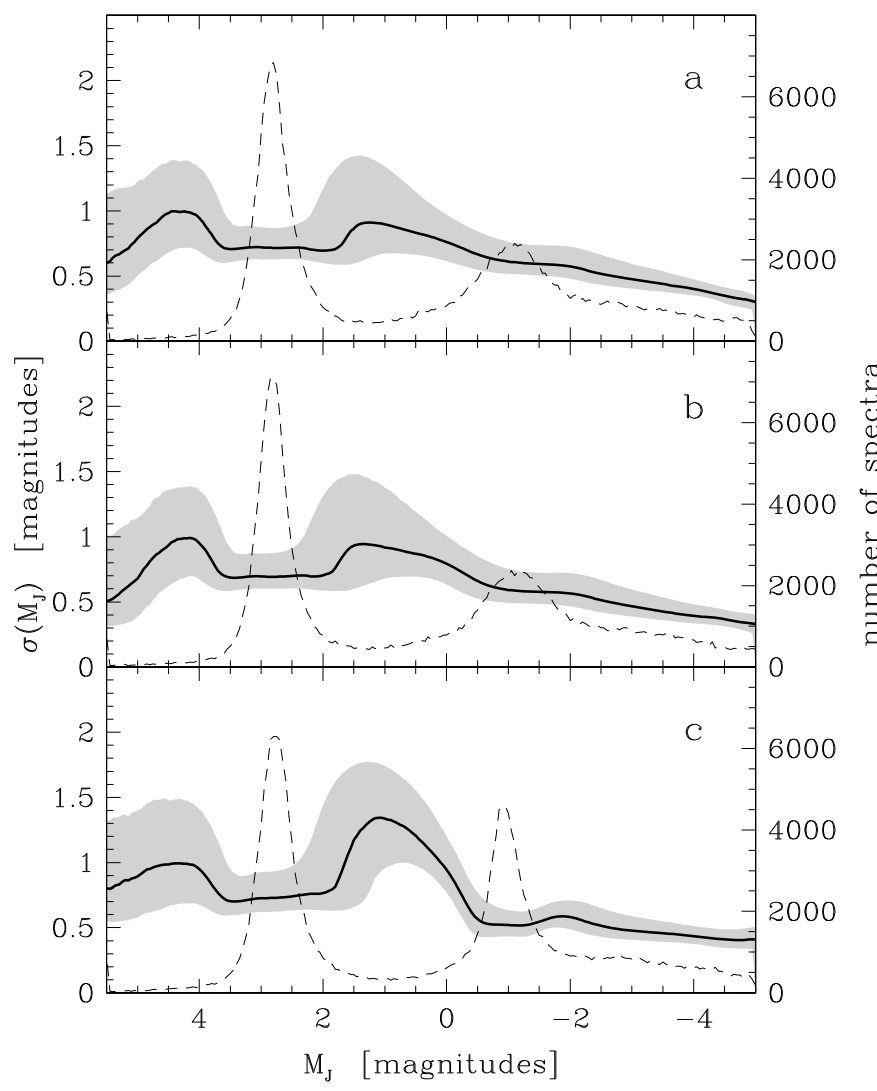

Fig. 8. Error in absolute magnitude as a function of absolute $J$ magnitude. The thick lines show running median errors for calculations using YY a), Dartmouth b) and Padova isochrones c). The Boxcar for the running median is $\pm 0.5 \mathrm{mag}$. The shaded area shows the 20 to 80 percentile range of the error distribution and the dashed line traces the number of objects per 0.1 mag wide absolute magnitude bin.

\subsection{Errors of derived distances from repeated observations}

Most of the RAVE objects are observed only once. But $\sim 10 \%$ of the observing time is devoted to repeated observations. This allows another check of the derived distance errors. Multiple observations of the same object are treated independently in our calculations, so the results can be directly compared. Note that repeated observations allow for a very efficient testing of the distance scatter, but they cannot point to possible systematic offsets of the mean. We believe that tests using Hipparcos stars with known parallaxes and observations of stars in clusters (Sect. 3) give sufficient confidence that any such offsets are insignificant for our results.

The total number of repeated objects in our sample is 19714 for which 50149 spectra have been obtained. This list is limited to objects with identical 2MASS identifiers for which RAVE has at least two spectra with $S / N>20$ and which do not show any peculiarities. Figure 10 plots the scatter of repeated observations in absolute magnitude and distance. The presentation follows that of Fig. 7. The results from repeated observations point to a remarkable repeatability of our distance estimates. Half of the objects show a distance scatter of less than $\simeq 11 \%$, and in $80 \%$ of the cases the distance scatter is smaller than $\simeq 31 \%$. Repeated observations have a similar distribution in $S / N$ as the other observations, with values which are only slightly higher. So Fig. 7 is representative also of the formal errors of repeated observations. Comparison of Figs. 7 and 10 suggests that our formal error estimates are rather conservative.

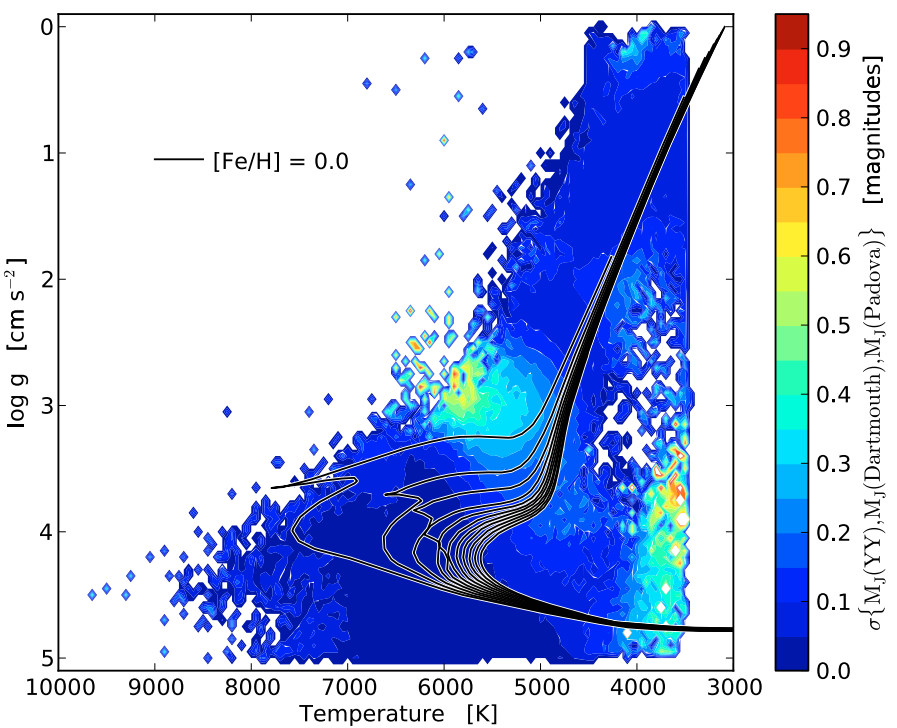

Fig. 9. Comparison of absolute $J$ magnitudes derived using either YY, Dartmouth, or Padova isochrone sets. Colors label an average dispersion in magnitudes for non-peculiar spectra with $S / N>20$ in a given (temperature, gravity) bin. Solar composition isochrones from the YY set with ages between 1 and 11 Gyr and with a 1 Gyr spacing are overlayed. Metal-poor isochrone sets would be shifted towards higher temperatures (see Fig. 2a).
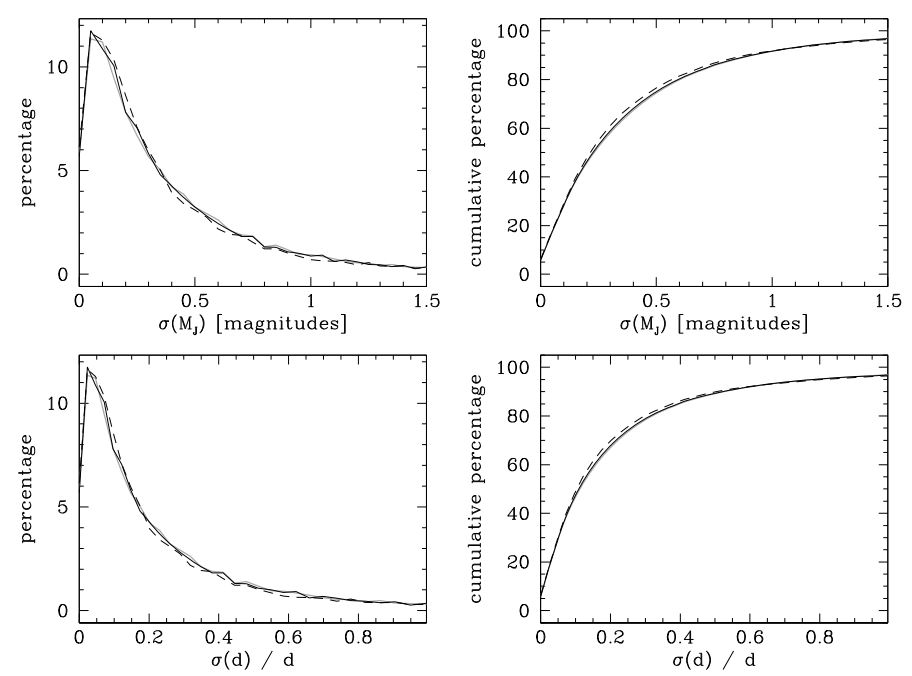

Fig. 10. Scatter of repeated observations of the same object. Scatter distribution histogram (left) and cumulative plot (right) for absolute $J$ magnitude (top) and distance (bottom). The number of repeated objects with distances calculated with a given set of isochrones is 19714 . The presentation follows the scheme of Fig. 7.

The scatter of distances derived from repeated observations depends on the type of star considered. We plot the scatter of repeated observations in Fig. 11, using the absolute $J$ magnitude as its label, in a manner similar to Fig. 8 for the formal errors. The morphological properties of the scatter of repeated observations is similar to the formal errors, but the values involved are much smaller, except for stars with $M_{J} \simeq 1.0$, where the scatters are similar. The median error for Solar type dwarfs $\left(M_{J} \simeq 3.0\right)$ is $\lesssim 0.2 \mathrm{mag}$. A similarly low scatter is reached also for red clump stars when using the Padova isochrones. The dashed lines in Fig. 11 marking the number of repeated objects closely follow the distribution of the whole sample (Fig. 8). Again they peak in the regions of the smallest errors. 


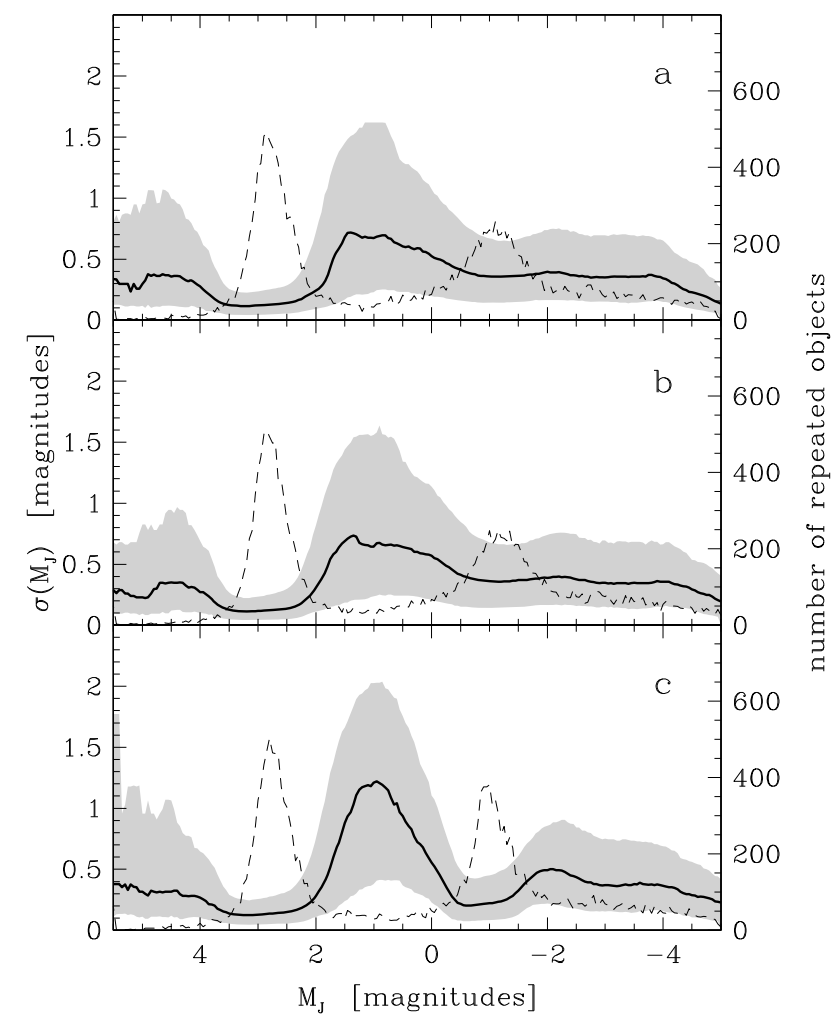

Fig. 11. Scatter of absolute magnitudes calculated for repeated observations of the same object as a function of absolute $J$ magnitude. Thick lines show running median errors for calculations using YY a), Dartmouth b) and Padova isochrones c). The Boxcar for the running median is $\pm 0.5 \mathrm{mag}$. The shaded area shows the 20 to 80 percentile range of the error distribution and the dashed line traces the number of repeated objects per 0.1 mag wide absolute magnitude bin.

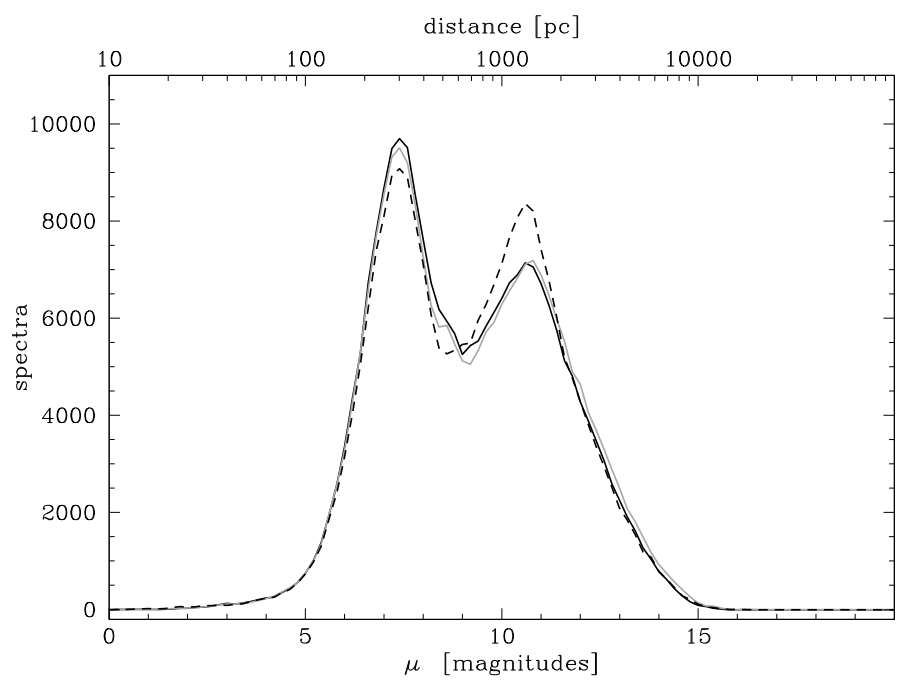

Fig. 12. Distribution of distance moduli $(\mu)$ for the three isochrone sets: YY (black), Dartmouth (grey), and Padova (dashed line). The bin size is 0.2 magnitudes.

\subsection{Distance distribution}

Figure 12 shows a distribution of distance moduli $\mu$. The results for YY and Dartmouth isochrones are very similar. The two peaks correspond to dwarfs at distances of $\sim 300 \mathrm{pc}$ and giants at $\sim 1.5 \mathrm{kpc}$, with some stars being as far away as $10 \mathrm{kpc}$. Calculations using Padova isochrones find more giants and their
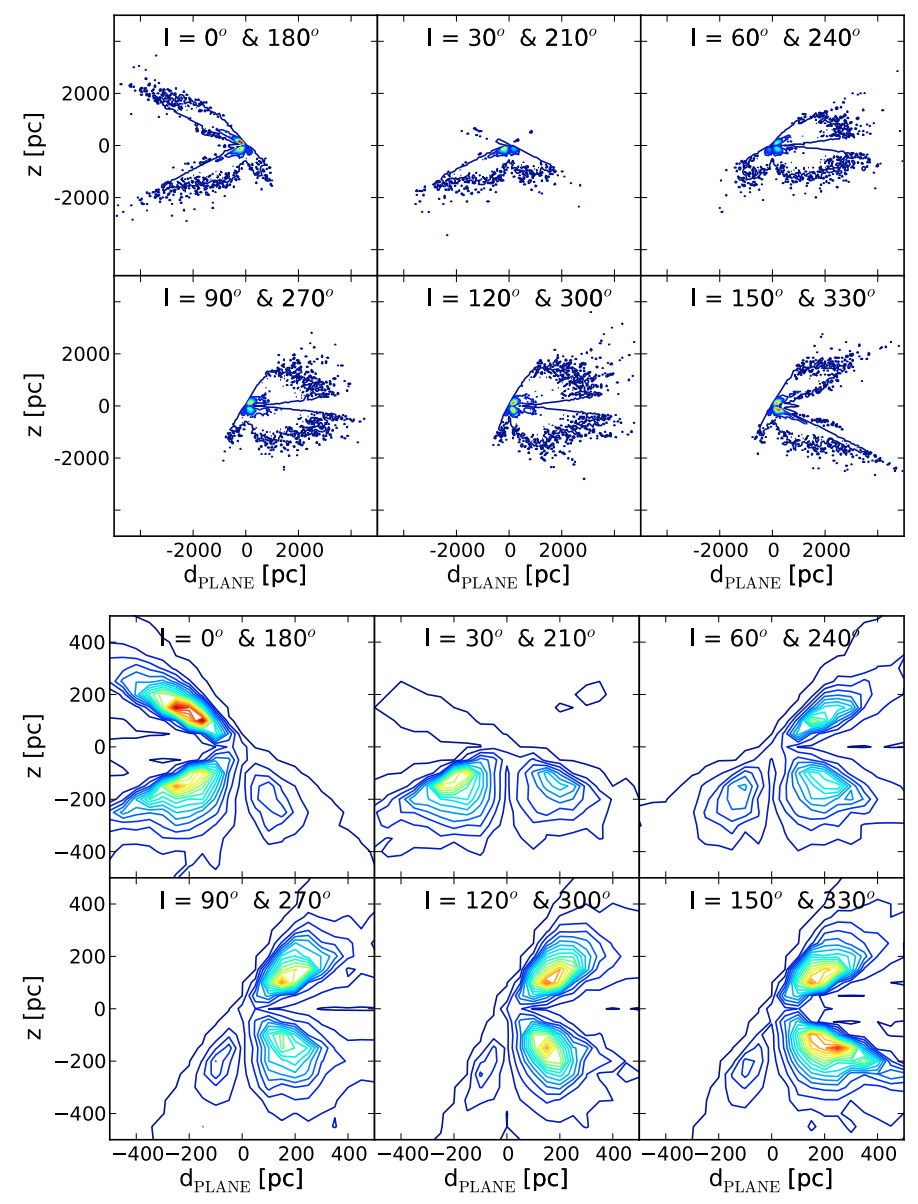

Fig. 13. Galactic vertical cross-sections of the sample for distances derived using the Padova isochrone set. Each plot contains stars with Galactic longitudes within $15^{\circ}$ of the labelled values. $d_{\text {PLANE }}$ is the cylindrical projected distance along the Galactic plane with negative values for stars within the Solar circle. $z$ is the distance from the Galactic plane. The density contours mark linearly spaced surface densities between 0.001 and $0.3 \mathrm{stars} / \mathrm{pc}^{2}$. The upper panel shows the whole sample of 235064 stars and the bottom panel shows an enlargement of the densest parts around the Galactic midplane.

distribution is more peaked. The difference is due to the redclump evolutionary phase which is present only in this isochrone set.

The observed sample, which is limited to the Southern Hemisphere, is influenced by the Galactic structure. This causes a directional dependence of the derived distances. B10 plot the projections of the sample to the Galactic $(x, y, z)$ coordinates (see Fig. 10 in B10). Here we make a more detailed plot (Fig. 13), dividing the sample into $30^{\circ}$-wide slices perpendicular to the Galactic plane. The upper set of graphs depicts the general situation, and the bottom one is zoomed-in by a factor of 10 . The Sun is located at the origin of the coordinate system, so its distance from the Galactic plane is neglected. About half of each graph is empty because we are limited to the southern skies. Also the general direction of the Southern galactic pole has been observed infrequently. This can be seen also in Fig. 21 of Z08.

The zoomed-in set in Fig. 13 reveals an expected property. Many of the isodensity contours do not follow circular arcs around the Solar position. They tend to be horizontal, i.e. parallel to the Galactic plane. This is expected and is a direct consequence of us living in a disky galaxy. But the fact that we can see it in Fig. 13 supports the notion that the measured distances are rather accurate. 
T. Zwitter et al.: Distance determination for RAVE stars using stellar models. II.

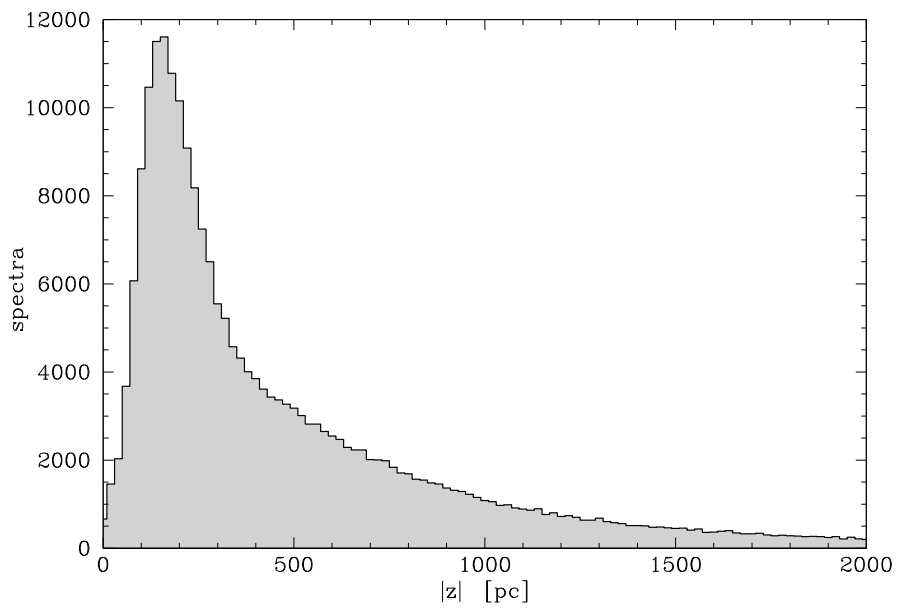

Fig. 14. Histogram of distances from the Galactic plane if the Sun is assumed to be very close to it. The distances are based on the Padova isochrone set.

Motivated by this result we plot the histogram of distances from the Galactic plane (Fig. 14), again neglecting the Solar displacement off the plane. The result seems rather important. The RAVE sample generally avoids regions within $\sim 20^{\circ}$ of the Galactic plane. So the histogram values increase until we hit the edge of the disk. The decreasing histogram values for stars with $|z|>200$ pc are well represented by two exponential components, one of them with a very small scale-height. We believe such properties illustrate the capacity of RAVE to address the issues of Galactic structure, using its measurements of kinematics, temperature, gravity, metallicity, and now distance. But to draw secure conclusions about the properties of the thin and thick disks, even if immediately suggestive by the double exponent in Fig. 14, requires further study. In particular, a detailed assessment of possible selection effects and a comparison to models of the Galactic structure is needed. Therefore a complete discussion is deferred to a separate paper.

\subsection{The Catalog}

The final catalog contains distances for 235064 spectra of 210.872 different stars. These are RAVE spectra with spectroscopically determined values of radial velocity, surface temperature, gravity and metallicity. Furthermore these spectra have $S / N>20$ and were not identified as peculiar by the automated pipeline.

A detailed description of the catalog is given in Table 3. Note that distances are given in distance moduli $(\mu)$ and not as a plain distance $(d)$. The reason is a nicer behaviour of errorbars. Errors of distance moduli are usually symmetric, while the corresponding distance errors are not. The catalog entries will be made publicly available through the CDS service alongside the regular RAVE public data releases. Currently the latest is the second data release (Z08) with the third one close to publication (Siebert et al. 2010). Distances to RAVE stars are derived from values of stellar parameters and their adopted errors. Should these change in the future we will recalculate the distance set and report on the results by updating the corresponding astro-ph and CDS entries.

\section{Discussion and conclusions}

The paper uses spectroscopically determined values of stellar parameters and the assumption that the star is a single object undergoing normal stellar evolution to determine its most likely distance. So the results are expected to be very useful for normal stars, while the values for peculiar objects (emission line stars, double line spectroscopic binaries) may suffer from systematic problems. We note that peculiar objects present a minority in our sample, and we also try to detect them using an automated classification pipeline. The pipeline is conservative, still it classified 235064 out of a total of 251364 spectra with $S / N>20$ as normal, i.e. a fraction of $93.5 \%$. Only the distances to such stars are published.

The present effort draws from the extensive experience gained during our initial work on the problem (B10). The method is similar but we introduce some refinements. First we note that isochrones calculated by different groups are not identical. So we give the distances using three recently published isochrone sets. The sets are those of Yonsei-Yale (Demarque et al. 2004), Dartmouth (Dotter et al. 2008), and Padova (Bertelli et al. 2008). This leaves it to the discretion of the user to choose the isochrone set that is best suited to a particular problem. Next we make sure that isochrones are spaced linearly in time and locally weighted so that the prevalence of low-mass stars is taken into account (we showed that the last point is not crucial for the results we get). Also, we take into account that the errors of stellar parameter values vary across the parameter space. We confirm that the error values published in the latest RAVE data release are rather conservative, still we prefer to be careful and avoid more optimistic error estimates as suggested by the repeated observations.

The essential differences between our method and that of B10 is this. B10 jittered the data by the observational errors and sought the model star that most closely matched each jittered data point, and then averaged the individual distances obtained in this way. We calculate for every model star the probability that it could give rise to the data, given the known observational errors. Then we use these weights to average the fluxes of the model stars and calculate the absolute magnitude of the star. By using the closest match, the B10 scheme gives a large weight to rapid phases of stellar evolution which sweep the otherwise empty parts of the (temperature, gravity) plane. Here we assume that stars are observed at a random point of their lives, that less massive stars are more numerous, and that higher luminosity ones are surveyed in a larger volume of space.

The results have been tested using two sets of stars for which the distances are known a priori. These are Hipparcos stars with well determined values of the trigonometric parallax, and stars that are secure members of stellar clusters. Distances determined from RAVE spectra match the astrometric values to within $\$ 22 \%$, with a mean difference of less than $3 \%$. This is similar to the results already obtained by B10. Hipparcos stars with well determined values of astrometric distance are almost exclusively dwarfs. So we need another set to check the distances derived for giant stars, which represent about half of the RAVE sample. We used members of open and globular clusters observed by RAVE. We identified spectra of 12 stars that are certain members of 7 open clusters and one globular cluster. We note all isochrone sets yield distances which agree with cluster distances to within $10 \%$ on average. But only the Padova isochrone set includes the red-clump phase, so the distances derived using the Padova set are the preferred ones for giant stars. The results for individual stars show a scatter of $20 \%$ with respect to the published cluster distances. This is encouraging, as it shows that distances derived spectroscopically agree well with other methods up to a range of several kiloparsecs. 
A\&A 522, A54 (2010)

Table 3. Description of the catalog of spectroscopic distances, available at the CDS.

\begin{tabular}{|c|c|c|c|c|c|}
\hline $\begin{array}{l}\text { Column } \\
\text { number }\end{array}$ & Character & Format & Acronym & Units & Description \\
\hline 1 & $1-17$ & $17 \mathrm{~S}$ & Object-id & & Object name \\
\hline 2 & 19-30 & $12.8 \mathrm{~F}$ & RA & degrees & Right Ascension (J2000.0) \\
\hline 3 & $32-43$ & $12.8 \mathrm{~F}$ & DE & degrees & Declination (J2000.0) \\
\hline 4 & $44-50$ & $6.1 \mathrm{~F}$ & RV & $\mathrm{km} \mathrm{s}^{-1}$ & Heliocentric radial velocity \\
\hline 5 & $52-57$ & $6.1 \mathrm{~F}$ & eRV & $\mathrm{km} \mathrm{s}^{-1}$ & Formal RV error \\
\hline 6 & $59-64$ & $6.1 \mathrm{~F}$ & pmRA & mas $\mathrm{yr}^{-1}$ & Proper motion in $\mathrm{RA}^{1}$ \\
\hline 7 & $66-71$ & $6.1 \mathrm{~F}$ & epmRA & mas $\mathrm{yr}^{-1}$ & Error in $\mathrm{pmRA}^{1}$ \\
\hline 8 & $73-78$ & $6.1 \mathrm{~F}$ & pmDE & mas $\mathrm{yr}^{-1}$ & Proper motion in $\mathrm{DE}^{1}$ \\
\hline 9 & $80-85$ & $6.1 \mathrm{~F}$ & epmDE & mas $\mathrm{yr}^{-1}$ & Error in $\mathrm{pmDE}^{1}$ \\
\hline 10 & 87-92 & $6.3 \mathrm{~F}$ & $J$ mag & mag & $J_{2 \text { MASS }}$ apparent magnitude \\
\hline 11 & 94-99 & $6.3 \mathrm{~F}$ & Kmag & mag & $K_{2 \text { MASS }}$ apparent magnitude \\
\hline 12 & 101-108 & $8 \mathrm{~S}$ & Obsdate & yyyymmdd & Date of observation \\
\hline 13 & $110-119$ & $10 \mathrm{~S}$ & Fieldname & & Name of the field \\
\hline 14 & $121-123$ & $3 \mathrm{G}$ & FibNum & & Fiber number \\
\hline 15 & $125-130$ & $6 \mathrm{G}$ & $T_{\text {eff }}$ & $\mathrm{K}$ & Effective temperature \\
\hline 16 & $132-136$ & $5.2 \mathrm{~F}$ & $\log g$ & $\log \left(\mathrm{cm} \mathrm{s}^{-2}\right)$ & Surface gravity \\
\hline 17 & $138-142$ & $5.2 \mathrm{~F}$ & {$[\mathrm{M} / \mathrm{H}]$} & & Metallicity \\
\hline 18 & $144-149$ & $6.2 \mathrm{~F}$ & $S / N$ & & Signal to noise per pixel \\
\hline 19 & $151-157$ & $7.3 \mathrm{~F}$ & $\mu(\mathrm{YY})$ & mag & Distance modulus (YY isochrones) \\
\hline 20 & 159-165 & $7.3 \mathrm{~F}$ & $\mathrm{e} \mu(\mathrm{YY})$ & mag & Formal error in $\mu(\mathrm{YY})$ \\
\hline 21 & $167-173$ & $7.3 \mathrm{~F}$ & $\mu($ Dart $)$ & mag & Distance modulus (Dartmouth isochrones) \\
\hline 22 & 175-181 & $7.3 \mathrm{~F}$ & $\mathrm{e} \mu($ Dart $)$ & mag & Formal error in $\mu$ (Dart) \\
\hline 23 & 183-188 & $7.3 \mathrm{~F}$ & $\mu$ (Padova) & mag & Distance modulus (Padova isochrones) \\
\hline 24 & 190-196 & $7.3 \mathrm{~F}$ & $\mathrm{e} \mu$ (Padova) & mag & Formal error in $\mu$ (Padova) \\
\hline
\end{tabular}

Notes. ${ }^{1}$ If the item is not available its value is set to $9.99 \mathrm{E} 8$.

The $H-R$ diagram using the derived distances now appears rather clean, with two well defined groups of stars that present the majority of the RAVE sample (Fig. 5). These are Solar-like dwarfs with $T_{\text {eff }} \sim 6000 \mathrm{~K}$ and giants with $T_{\text {eff }} \sim 4750 \mathrm{~K}$. About half of the latter are members of the red clump, with the rest being on the red-giant branch. We derive the absolute magnitude of the red clump as $M_{J}=-0.91 \pm 0.02$ and $M_{K}=-1.61 \pm 0.02$ which is consistent with the values from the literature.

Distance errors have been estimated internally within the method and by using repeated observations of the same object. The internal estimates appear to be rather conservative and give median errors on distance of $33 \%$. The repeatability of derived distances is much better: the distances derived from individual spectra of the same object match at a level of $11 \%$.

Derived distances are based on apparent magnitudes in the $J_{2 \text { MASS }}$ band. We assume that interstellar reddening is negligible because the vast majority of RAVE stars lie at high Galactic latitudes $\left(|b|>20^{\circ}\right)$. Nevertheless it is interesting to compare the $J_{2 \text { MASS }}$ results with the ones derived using the $K_{2 \text { MASS }}$ band, which is even redder and so even less sensitive to reddening. So we calculated the distances to the whole sample using the $K_{2 \mathrm{MASS}}$ bandpass and the Padova isochrone set. Figure $15 \mathrm{com}-$ pares the results for both bandpasses. The average distance ratio is $\overline{d_{K} / d_{J}}=0.983$ and the scatter is $\sigma\left(d_{K} / d_{J}\right)=0.039$. Only $5 \%$ of the spectra have $d_{K} / d_{J}<0.92$ and $5 \%$ of the spectra have $d_{K} / d_{J}>1.04$. Comparison of our distances and the Hipparcos trigonometric parallax determinations (Sect. 3.1) gives similar results for both bands, with the scatter in the distance ratio being 0.25 for the $J$ band and 0.24 for the $K$ band. Also the results for stellar clusters are very similar. The scatter of spectroscopic distances around the cluster distances from the literature is $22 \%$ for the $J$ band and $23 \%$ for the $K$ band.

One can test whether about $2 \%$ smaller distances derived from the $K$ instead of the $J$ band are consistent with a decreased

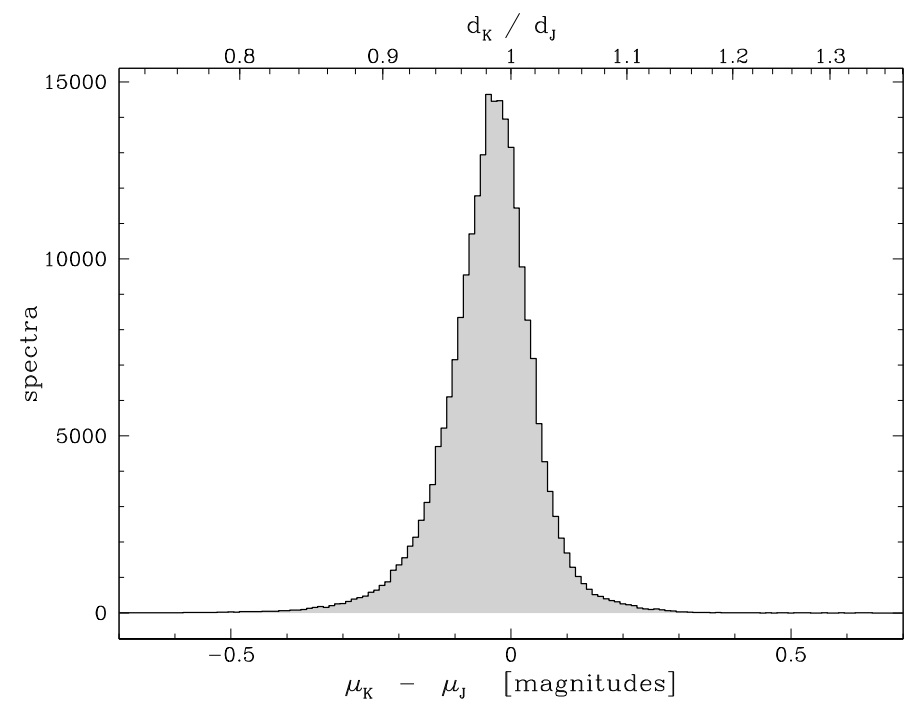

Fig. 15. Comparison of distances (top axis) and distance moduli (bottom axis) for a calculation with Padova isochrones if using the $J_{2 \text { MASS }}$ or $K_{2 \text { MASS }}$ magnitudes.

influence of interstellar reddening at longer wavelengths. From relations for extinction for the Solar energy distribution $\left(A_{J} \simeq\right.$ $0.887 E_{B-V}$ and $A_{K} \simeq 0.382 E_{B-V}$, Fiorucci \& Munari 2003) and from $\overline{A_{K}-A_{J}} \simeq 5 \log (0.983)$ we get $\overline{E_{B-V}} \simeq 0.07$. This is larger than the values of integrated colour excess toward the South Galactic pole which range from $E_{B-V}=0.018$ (Schlegel et al. 1998) to $E_{B-V}=0.04$ (Teerikorpi 1990). The RAVE sample has $\overline{\csc |b|}=3.22$, where $b$ is the Galactic lattitude. So the difference between our average color excess and the values at the South Galactic pole can be explained with a simple slab model of the Galactic disk. 
We note that a comparison of distances derived using different passbands can improve our understanding of reddening at high Galactic lattitudes. This will be discussed in a separate paper, where we shall also address the rare cases of large differences in the derived distances which may point to spectral peculiarities. The photometric effect of interstellar reddening can then be confirmed spectroscopically from RAVE data by measurement of the equivalent width of the diffuse interstellar band at $8620 \AA$ (Munari et al. 2008). We conclude that distances derived using either the $J$ or the $K$ band are very similar, but a bit smaller measurement errors of apparent magnitudes favour the $J$ band as our baseline.

The main result of this paper is a catalog of newly derived stellar distances. The paper discusses distances to 235064 stars. The list will increase with time as the RAVE survey progresses. The public version of the catalog will be accessible through the CDS service, with its size growing along with the public RAVE data releases. Note that this is not the only current effort to derive distances to RAVE stars. Our results are based on the work of B10, and there is also a separate project of distance derivation using a Bayesian approach (Burnett \& Binney 2010). We believe the availability of complementary computations will be of assistance to users, who can then select those best suited to their particular problem.

The present results place RAVE dwarfs at typical distances of $\sim 300 \mathrm{pc}$, and giants at 1 or $2 \mathrm{kpc}$, even though there are objects $10 \mathrm{kpc}$ away. In fact RAVE reached even the brightest LMC stars (Munari et al. 2009). So the RAVE survey is well placed between the more local Geneva Copenhagen Survey (GCS, Nordström et al. 2004; Holmberg et al. 2009) and the stellar component (SEGUE) of the Sloan Digital Sky Survey (Abazajian et al. 2009). Two thirds of the GCS stars are closer than $100 \mathrm{pc}$ and $95.5 \%$ are closer than $200 \mathrm{pc}$ from the Sun, while most of the SEGUE stars are members of the halo. For example the full sample of SDSS/SEGUE DR-7 calibration stars contains only $\sim 2400$ stars within $1 \mathrm{kpc}$ from the Galactic plane (Beers 2009). These results make the RAVE survey ideal to study the properties of the Galactic thin and thick disks. The flatness of the stellar distribution can be seen also directly from the shape of the isodensity contours in cross-sections perpendicular to the galactic plane. This confirms the capability of the RAVE survey to address some of the most fundamental questions of Galactic astrophysics. The possibility to upgrade the RAVE metallicity measurements with individual element abundances (Boeche et al. 2010) only reinforces such a conclusion. Individual applications of this dataset with full 6-dimensional kinematical and physical information will be discussed in separate papers.

Acknowledgements. Funding for RAVE has been provided by: the AngloAustralian Observatory; the Astrophysical Institute Potsdam; the Australian National University; the Australian Research Council; the French National Research Agency; the German Research foundation; the Istituto Nazionale di Astrofisica at Padova; The Johns Hopkins University; the National Science Foundation of the USA (AST-0908326); the W. M. Keck foundation; the Macquarie University; the Netherlands Research School for Astronomy; the Natural Sciences and Engineering Research Council of Canada; the Slovenian Research Agency; the Swiss National Science Foundation; the Science \& Technology Facilities Council of the UK; Opticon; Strasbourg Observatory; and the Universities of Groningen, Heidelberg and Sydney. The RAVE web site is at http: //www . rave-survey . org.

Note added in proof New values of stellar parameters became available after acceptance of the paper. The original results are based on the parameter determination pipeline as described in Zwitter et al. (2008), while the derivation of new values is explained in Siebert et al. (2010).
Another recent progress is a redetermination of errors of stellar parameters across the H-R diagram. Instead of the conservative external errors reported in Zwitter et al. (2008) we could use an extensive RAVE subset where a certain star was observed more than once. Errors on stellar parameters were estimated from the scatter of independent parameter determinations for a given star and averaged over stars in the given bin in temperature and gravity (Siebert et al. 2010). This internal error seems trustworthy, as there are no appreciable zero-point offsets between our stellar parameter values and those of the reference datasets (see Table 5 in Zwitter et al. 2008). The distance moduli have been calculated for the Padova isochrone set using the new values of stellar parameters. The assumed errors were either the conservative values used in the accepted version of the paper or the ones determined from repeated observations. Both distance moduli and their errors add four columns to the catalog of spectroscopic distances, available via the CDS. New values of stellar parameters yield distances which are generally closer to the reference values than the ones reported in the main paper. The scatter $(\sigma)$ of the spectroscopic $(d)$ to trigonometric $\left(d_{\varpi}\right)$ distance ratio is 0.28 and 0.22 if the external and internal error estimates are used, respectively. This test was done on the same sample of Hipparcos stars as the one reported in Table 1 where the corresponding scatter equals 0.25 . A similar test can be performed using members of star clusters. The scatter of the spectroscopic to cluster distance ratio is 0.29 for the distance calculations using Padova isochrones and reported in the main paper. The new values of stellar parameters diminish the scatter to 0.205 . This value is obtained for both choices of error estimation: the conservative external error estimates and the internal ones obtained from repeated observations. We conclude that the best match between our spectroscopic distances and the reference values is obtained when using the Padova isochrone set, new values of stellar parameters and errors determined from repeated observations.

\section{References}

Abazajian, K. N., Adelman-McCarthy, J. K., Agüeros, M. A., et al. 2009, ApJS, 182,543

Beers, T., 2009, in Proc. IAU Symp., 265, 453

Bertelli, G., Girardi, L., Marigo, P., et al. 2008, A\&A, 484, 815

Boeche, C., Siebert, A., Steinmetz, M., et al. 2010, BAAS, 41, 479

Breddels, M. A., Smith, M. C., Helmi, A., et al. 2010, A\&A, 511, A16 (B10, Paper I)

Burnett, B., Binney, J. 2010, MNRAS, 407, 339

Chabrier, G. 2003, PASP, 115, 763

Demarque, P., Woo, J.-H., Kim, Y.-C., \& Yi, S. K. 2004, ApJS, 155, 667

Dias, W. S., Alessi, B. S., Moitinho, A., \& Lepine, J. R. D. 2002, A\&A, 389, 871

Dotter, A., Chaboyer, B., Jevremović, D., et al. 2008, ApJS, 178, 89

Fiorucci, M., \& Munari, U. 2003, A\&A, 401, 781

Freeman, K.C., \& Bland-Hawthorn, J. 2002. ARA\&A, 40487

Groenewegen, M. A. T. 2008, A\&A, 488, 935

Harris, W. E. 1996, AJ, 112, 1487 (rev. 15 May 1997)

Holmberg, J., Nordström, B., \& Andersen, J., 2009, A\&A, 501, 941

Klement, R., Fuchs, B., \& Rix, H.-W. 2008, ApJ, 685, 261

Koen, C., Marang, F., Kilkenny, D., \& Jacobs, C. 2007, MNRAS, 380, 1433

Matijevič, G., Zwitter, T., Munari, U., et al. 2010, AJ, 140, 184

Munari, U., Sordo, R., Castelli, F., \& Zwitter, T. 2005, A\&A, 442, 615

Munari, U., Tomasella, L., Fiorucci, M., et al. 2008, A\&A, 488, 969

Munari, U., Siviero, A., Bienaymé, O., et al. 2009, A\&A, 503, 511

Nordström, B., Mayor, M., Andersen, J., et al. 2004, A\&A, 418, 989

Schlegel, D. J., Finkbeiner, D. P., \& Davis, M. 1998, ApJ, 500, 525

Seabroke, G. M., Gilmore, G., Siebert, A., et al. 2008, MNRAS, 384, 11

Siebert, A., Bienaymé, O., Binney, J., et al. 2008, MNRAS, 391, 793

Siebert, A., et al. 2010, AJ, accepted

Smith, M. C., Ruchti, G. R., Helmi, A., et al. 2007, MNRAS, 379, 755

Steinmetz, M. 2003, ASP Conf. Ser., 298, 381

Steinmetz, M., Zwitter, T., Siebert, A., et al. 2006, AJ, 132, 1645

Steinmetz, M., Siebert, A., Zwitter, T., et al. 2009, in The Galaxy Disk in Cosmological Context, Proc. IAU Symp., 254, 453

Teerikorpi, P. 1990, A\&A, 235, 362

Valentini, M., \& Munari, U. 2010, A\&A, in press, [arXiv: 1007.0207]

Veltz, L., Bienaymé, O., Freeman, K. C., et al. 2008, A\&A, 480, 753

Van Helshoecht, V., \& Groenewegen, M. A. T. 2007, A\&A, 463, 559

van Leeuwen, F. 2007, Astrophysics and Space Science Library, 350

Zwitter, T., Siebert, A., Munari, U., et al. 2008, AJ, 136, 421 (Z08) 